\title{
Loss of Piccolo Function in Rats Induces Cerebellar Network Dysfunction and Pontocerebellar Hypoplasia Type 3-like Phenotypes
}

\author{
Joanne Falck, ${ }^{1}$ Christine Bruns, ${ }^{1}$ Sheila Hoffmann-Conaway, ${ }^{1}$ Isabelle Straub, ${ }^{2}$ Erik J. Plautz, ${ }^{3}$ Marta Orlando, ${ }^{4}$ \\ Humaira Munawar, ${ }^{5}$ Marion Rivalan, ${ }^{4,5}$ York Winter, ${ }^{4,5}$ Zsuzsanna Izsvák, ${ }^{6}$ Dietmar Schmitz, ${ }^{4}$ F. Kent Hamra, \\ Stefan Hallermann, ${ }^{2}$ Craig Curtis Garner, ${ }^{1 *}$ and $\odot$ Frauke Ackermann ${ }^{1 *}$ \\ ${ }^{1}$ German Center for Neurodegenerative Diseases, Charité Medical University, 10117 Berlin, Germany, ${ }^{2}$ Carl-Ludwig Institute for Physiology, 04103 Leipzig, \\ Germany, ${ }^{3}$ Department of Neurology and Neurotherapeutics, University of Texas Southwestern, Dallas, Texas $75390,{ }^{4}$ Charité Medical University Berlin and \\ Berlin Institute of Health, NeuroCure Cluster of Excellence, 10117 Berlin, Germany, ${ }^{5}$ Department of Biology, Humboldt University, 10099 Berlin, Germany, \\ ${ }^{6}$ Max Delbrück Center for Molecular Medicine in the Helmholtz Society, 13125 Berlin, Germany, and ${ }^{7}$ Department of Obstetrics and Gynecology, Cecil H. \\ and Ida Green Center for Reproductive Biology Sciences, University of Texas Southwestern, Dallas, Texas 75390
}

Piccolo, a presynaptic active zone protein, is best known for its role in the regulated assembly and function of vertebrate synapses. Genetic studies suggest a further link to several psychiatric disorders as well as Pontocerebellar Hypoplasia type 3 (PCH3). We have characterized recently generated Piccolo KO $\left(P c l 0^{g t / g t}\right)$ rats. Analysis of rats of both sexes revealed a dramatic reduction in brain size compared with WT $\left(P c l{ }^{w t / w t}\right)$ animals, attributed to a decrease in the size of the cerebral cortical, cerebellar, and pontine regions. Analysis of the cerebellum and brainstem revealed a reduced granule cell layer and a reduction in size of pontine nuclei. Moreover, the maturation of mossy fiber afferents from pontine neurons and the expression of the $\alpha 6 \mathrm{GABA}_{\mathrm{A}}$ receptor subunit at the mossy fiber-granule cell synapse are perturbed, as well as the innervation of Purkinje cells by cerebellar climbing fibers. Ultrastructural and functional studies revealed a reduced size of mossy fiber boutons, with fewer synaptic vesicles and altered synaptic transmission. These data imply that Piccolo is required for the normal development, maturation, and function of neuronal networks formed between the brainstem and cerebellum. Consistently, behavioral studies demonstrated that adult $P c l o^{g t / g t}$ rats display impaired motor coordination, despite adequate performance in tasks that reflect muscle strength and locomotion. Together, these data suggest that loss of Piccolo function in patients with $\mathrm{PCH} 3$ could be involved in many of the observed anatomical and behavioral symptoms, and that the further analysis of these animals could provide fundamental mechanistic insights into this devastating disorder.

Key words: active zone; Piccolo; pontocerebellar hypoplasia; presynapse

Significance Statement

Pontocerebellar Hypoplasia Type 3 is a devastating developmental disorder associated with severe developmental delay, progressive microcephaly with brachycephaly, optic atrophy, seizures, and hypertonia with hyperreflexia. Recent genetic studies have identified non-sense mutations in the coding region of the PCLO gene, suggesting a functional link between this disorder and the presynaptic active zone. Our analysis of Piccolo KO rats supports this hypothesis, formally demonstrating that anatomical and behavioral phenotypes seen in patients with Pontocerebellar Hypoplasia Type 3 are also exhibited by these Piccolo deficient animals.

\section{Introduction}

Pontocerebellar hypoplasia is a rare and highly heterogeneous group of neurological disorders, often with a genetic origin, char-

Received Sept. 24, 2019; revised Feb. 10, 2020; accepted Feb. 13, 2020.

Author contributions: J.F., M.R., Y.W., Z.I., D.S., F.K.H., S.H., C.C.G., and F.A. designed research; J.F., C.B., I.S., E.J.P., M.O., H.M., and M.R. performed research; J.F., I.S., E.J.P., M.O., H.M., and M.R. analyzed data; J.F. wrote the first draft of the paper; J.F., S.H.-C., C.C.G., and F.A. edited the paper; J.F. and C.C.G. wrote the paper. acterized by an abnormally small cerebellum and pons (Rajab et al., 2003; Namavar et al., 2011). In Type 3 Pontocerebellar Hypoplasia (PCH3), also known as cerebellar atrophy with progres- 
sive microcephaly, patients suffer from severe developmental delay, progressive microcephaly with brachycephaly, seizures, hypertonia with hyperreflexia, and short stature (Rajab et al., 2003; Namavar et al., 2011). Additional features include the presence of craniofacial dysmorphisms and optic atrophy (Durmaz et al., 2009; Rudnik-Schöneborn et al., 2014).

Previous studies mapped the $\mathrm{PCH}$ phenotype to chromosome 7q11-21 (Rajab et al., 2003; Durmaz et al., 2009). More recently, a single nucleotide polymorphism in the human PCLO gene, indeed, located on chromosome 7 at position 21.11, has been found in patients with $\mathrm{PCH} 3$. This non-sense mutation is predicted to eliminate the $\mathrm{C}$ terminus of the longest Piccolo isoforms, including its PDZ and C2 domains (Ahmed et al., 2015), and perhaps destabilize the protein, leading to the hypothesis that Piccolo loss of function (LOF) is responsible for the phenotypes seen in this neurodevelopmental/neurodegenerative disorder.

Piccolo is a very large $(560 \mathrm{kDa})$ multidomain presynaptic scaffold protein and core component of the cytoskeletal matrix assembled at active zones (AZs) (Cases-Langhoff et al., 1996). A range of studies suggest that Piccolo uses its multidomain structure to scaffold not only other cytoskeletal matrix assembled at $\mathrm{AZ}$ proteins critical for the regulated release of neurotransmitters, but also proteins involved in the dynamic assembly of F-actin, synaptic vesicle (SV) recycling, and synapse integrity (Gundelfinger et al., 2015; Ackermann et al., 2019). Intriguingly, Piccolo is present at nearly every synaptic subtype, including glutamatergic, GABAergic, cholinergic, and dopaminergic synapses within the CNS and PNS (Cases-Langhoff et al., 1996; Fenster et al., 2000; Fenster and Garner, 2002) and is highly expressed in the cerebrum, hippocampus, cerebellum, and olfactory bulb, among others (Cases-Langhoff et al., 1996; Uhlén et al., 2015). It is one of the very first AZ proteins recruited to nascent synapses in vitro as well as in the developing brain (Zhai et al., 2001). For example, Piccolo appears at emerging synapses formed between mossy fiber (MF) boutons and cerebellar granule cells (GCs) as well as between parallel fiber (PF) and Purkinje cell (PC) synapses during the earliest stages of cerebellar development (Zhai et al., 2001). The large size of Piccolo and the complexity of the Pclo gene have thwarted most efforts to elucidate its function, although critical roles in retinal ribbon synapse formation and visual function (RegusLeidig et al., 2014; Müller et al., 2019) as well as the integrity of hippocampal synapses have been identified (Waites et al., 2013). What remains unclear is how Piccolo contributes to cerebellar development and whether, as suggested by genetic studies, it has a primary role in the etiology of $\mathrm{PCH}$. The recent generation of a Piccolo KO rat $\left(\mathrm{Pclo}^{\mathrm{gt} / \mathrm{gt}}\right)$ using transposon mutagenesis (Medrano et al., 2019) provides an opportunity to explore this potential relationship.

In the current study, we have assessed the contribution of Piccolo to cerebellar structure and function through the anatomical, functional, and behavioral characterization of adult $P \mathrm{clo}^{g t / g t}$ rats. Our analysis reveals a striking number of similarities to patients with $\mathrm{PCH} 3$, including a smaller cerebral cortex, a reduced volume of the cerebellum and pons, as well as impaired motor control and the presence of seizures. Our analysis has also uncov-

\footnotetext{
by the Neuro-Models Facility (E.J.P.) at the University of Texas Southwestern Medical Center, and supported by the Haggerty Center for Brain Injury and Repair. We thank Susanne Wegmann and Eckart Gundelfinger for discussion and valuable comments on the manuscript; and Anny Kretschmer and Katja Czieselsky for technical assistance. The authors declare no competing financial interests.

*C.C.G. and F.A. contributed equally to this work.

Correspondence should be addressed to Frauke Ackermann at Frauke.Ackermann@dzne.de.

https://doi.org/10.1523/JNEUROSCI.2316-19.2020

Copyright $\odot 2020$ the authors
}

ered changes in the anatomical and ultrastructural features of MF terminals and electrophysiological properties of these synapses. Together, these phenotypes are predicted to not only alter the functionality of the cerebellum but to contribute to motor and perhaps also behavioral dysfunctions seen in PCH3-affected individuals.

\section{Materials and Methods}

Generation of Piccolo KO rats (Pclo ${ }^{g t / g t}$ )

Generation of mutant Piccolo rat strains: Mutant rat strains harbored Sleeping Beauty $\beta$-Geo trap transposons (Ivics et al., 2009), originally transmitted from a donor, recombinant rat spermatogonial stem cell library (Izsvák et al., 2010). Recipient males were bred with WT females to produce a random panel of mutant rat strains enriched with gene traps in protein coding genes (Izsvák et al., 2010). All experiments were approved by the Institutional Animal Care and Use Committee at University of Texas Southwestern Medical Center in Dallas, as certified by the Association for Assessment and Accreditation of Laboratory Animal Care International NIH OLAW Assurance \#D16-00296.

\section{Characterization of pups and genotyping}

$\mathrm{P} 0-\mathrm{P} 2$ rats were weighed using Kern $440-43 \mathrm{~N}$ scales and measured for approximate length with a ruler. Genotyping of pups' cortices later revealed their genetic identity.

$\mathrm{P} 0-\mathrm{P} 2$ pups were genotyped using a PCR-based reaction. In brief, brain tissue was digested in lysis buffer ( $100 \mathrm{~mm}$ Tris- $\mathrm{HCl}$, pH 8.0, with $10 \mathrm{mg} / \mathrm{ml}$ proteinase $\mathrm{K}, 100 \mathrm{~mm} \mathrm{NaCl}$ ) for $5 \mathrm{~min}$ at $55^{\circ} \mathrm{C}$, before inhibiting proteinase $\mathrm{K}$ by incubation at $99^{\circ} \mathrm{C}$ for $10 \mathrm{~min}$. Samples were then centrifuged at $14,800 \mathrm{rpm}$ for $2 \mathrm{~min}$, and $1 \mu \mathrm{l}$ of supernatant was used for the PCR as outlined below.

For determination of genotype for adult rats, earpieces were taken and digested overnight at $55^{\circ} \mathrm{C}$ in SNET-buffer ( $400 \mathrm{~mm} \mathrm{NaCl}, 1 \%$ SDS, 200 $\mathrm{mm}$ Tris, $\mathrm{pH}$ 8.0, $5 \mathrm{~mm}$ EDTA) containing $10 \mathrm{mg} / \mathrm{ml}$ proteinase K. Proteinase $\mathrm{K}$ enzyme reaction was stopped, incubating the samples for $10 \mathrm{~min}$ at $99^{\circ} \mathrm{C}$. The mixture was centrifuged for $2 \mathrm{~min}$ at $14,800 \mathrm{rpm}$. Supernatant was transferred into a fresh tube, and DNA was precipitated by addition of $100 \%$ isopropanol. Then samples were centrifuged for 15 $\min$ at $4^{\circ} \mathrm{C}, 13,000 \mathrm{rpm}$. Precipitated DNA was washed once with $70 \%$ ethanol and centrifuged again for $5 \mathrm{~min}$ at 13,000 rpm. Supernatant was discarded, and the DNA pellet was air dried and resuspended in $\mathrm{H}_{2} \mathrm{O}$. A PCR with a specific primer combination was performed on isolated DNA. The following primers were used: Pclo KO F2: $3^{\prime}$-gcaggaacacaaac caacaa-5'; Pclo KO R1: 3' -tgacctttagccggaactgt-5'; SBF2: 3' -tcatcaaggaaa ccctggac- $5^{\prime}$. The PCR protocol was as follows: $2 \min 94^{\circ} \mathrm{C} ; 3 \times\left(30 \mathrm{~s} 94^{\circ} \mathrm{C}\right.$, $\left.60^{\circ} \mathrm{C} 30 \mathrm{~s}, 72^{\circ} \mathrm{C} 30 \mathrm{~s}\right) ; 35$ times $\left(94^{\circ} \mathrm{C} 30 \mathrm{~s}, 55^{\circ} \mathrm{C} 30 \mathrm{~s}, 72^{\circ} \mathrm{C} 30 \mathrm{~s}\right) ; 72^{\circ} \mathrm{C} 10$ $\mathrm{min}$. Samples were mixed with a loading dye (New England BioLabs) and run on $2 \%$ agarose gel (Serva) at $110 \mathrm{~V}$ for $45 \mathrm{~min}$. The gel was imaged using BioDocAnalyze UV transilluminator and BioDocAnalyze2.2 software.

\section{Western blot analysis}

Brains from $\mathrm{P} 0-\mathrm{P} 2$ pups were lysed in lysis buffer (50 mM Tris- $\mathrm{HCl}, 150$ mм NaCl, 5 mм EDTA, 1\% Triton X-100, 0.5\% deoxycholate, protease inhibitor $\mathrm{pH}$ 7.5) and incubated on ice for $5 \mathrm{~min}$. Samples were centrifuged at $13,000 \mathrm{rpm}$ for $10 \mathrm{~min}$ at $4^{\circ} \mathrm{C}$. Afterward the supernatant was transferred into a fresh tube, and the protein concentration was determined using a BCA protein assay kit (Thermo Fisher Scientific). The same protein amounts for $\mathrm{Pclo}^{w t / w t}, \mathrm{Pclo}^{w t / g t}$, and $\mathrm{Pclo}^{g t / g t}$ samples were separated by SDS-PAGE and transferred onto nitrocellulose membranes (running buffer: $25 \mathrm{~mm}$ Tris, $190 \mathrm{~mm}$ glycine, 0.1\% SDS, pH 8.3; transfer buffer: $25 \mathrm{~mm}$ Tris, $192 \mathrm{~mm}$ glycine, 1\% SDS, 10\% methanol for small proteins, $7 \%$ methanol for larger proteins, $\mathrm{pH} 8.3$ ). After the transfer, nitrocellulose membranes were blocked with $5 \%$ milk in TBST $(20 \mathrm{~mm}$ Tris, $\mathrm{pH} 7.5,150 \mathrm{~mm} \mathrm{NaCl}, 0.1 \%$ Tween 20 ) and incubated with primary antibodies in $3 \%$ milk in TBST overnight at $4^{\circ} \mathrm{C}$. Antibodies against Piccolo (1:1000; rabbit; Synaptic Systems; catalog \#142002) were incubated overnight. Nitrocellulose membranes were washed 3 times for 10 min with TBST and incubated with HRP-conjugated secondary antibodies for $1 \mathrm{~h}$ at room temperature (1:1000; Sigma Millipore; catalog \#NA934). Membranes were washed 3 times for 10 min with TBST; after- 
ward secondary antibody binding was detected with ECL Western Blotting Detection Reagents (Thermo Fisher Scientific) and a Fusion FX7 image and analytics system (Vilber Lourmat).

\section{Immunohistochemistry}

Immunohistochemistry was performed on brain tissue from rats perfused transcardially with 4\% PFA (Roth) dissolved in PB (80 mM $\mathrm{Na}_{2} \mathrm{HPO}_{4}$ (Roth), $20 \mathrm{~mm} \mathrm{NaH} \mathrm{PO}_{4}$ (Bernd Kraft) (PFA). Brains were extracted and placed in 4\% PFA overnight, cryoprotected in 15\% and then 30\% sucrose solution (Sigma Millipore; dissolved in PB) for $24 \mathrm{~h}$ each. Brains were snap-frozen by submersion in $-60^{\circ} \mathrm{C} 2$-methylbutane (Roth) and then stored at $-20^{\circ} \mathrm{C}$ until use. Brains were cut parasagittally or coronally with a Leica Microsystems cryostat to either 20- $\mu \mathrm{m}$-thick sections and mounted on Superfrost slides (Thermo Fisher Scientific), or 50 or $35 \mu \mathrm{m}$ sections, which were processed free-floating. Slides were left to dry for a minimum of $1 \mathrm{~h}$ before storage at $-20^{\circ} \mathrm{C}$, and free-float sections were stored in antifreeze solution: $30 \%$ ethylene glycol, $30 \%$ glycerol (Roth); $30 \%$ dd $\mathrm{H}_{2} \mathrm{O}, 10 \% 0.244 \mathrm{M} \mathrm{PO}_{4}$ buffer, $\mathrm{NaOH}, \mathrm{NaH}_{2} \mathrm{PO}_{4}$ (Roth) at $-20^{\circ} \mathrm{C}$.

Before staining, at least 4 slides (each containing two sections) from each animal were left to equilibrate at room temperature for $1 \mathrm{~h}$. Sections were selected to encompass the range of the axis we were investigating. A hydrophobic barrier was created around sections using a DAKO pen, and sections were washed and permeabilized with TBS (20 mM Tris, pH 7.5, $150 \mathrm{~mm} \mathrm{NaCl}$, Roth) with $0.025 \%$ Triton X-100 (Roth) (TBST) for $3 \times 5$ min, before blocking with $10 \%$ normal goat serum (Sigma Millipore) with $1 \%$ BSA (Sigma Millipore) in TBS. The following primary antibodies were used: calbindin (1:750; rabbit; Synaptic Systems; catalog \#214002), $\mathrm{GABA}_{\mathrm{A}}$ receptor $\alpha 6$ subunit (1:250; rabbit; Sigma Millipore; catalog \#G5544), MAP2 (1:1000; chicken; Sigma Millipore, catalog \#AB5543), Piccolo (1:200; guinea pig; Synaptic Systems; catalog \#142104), VGluT1 (1:1000; rabbit; Abcam; catalog \#ab104898), VGluT2 (1:250; guinea pig; Synaptic Systems; catalog \#13540419), and VGluT2 (1:300; mouse monoclonal [epitope AA 566-582]; Synaptic Systems; catalog \#135421). Antibodies diluted in TBS with 1\% BSA were applied and left overnight at $4^{\circ} \mathrm{C}$. After $3 \times 5$ min washing with TBST, differently labeled secondary antibodies were used from Invitrogen (Thermo Fisher Scientific, dilution $1: 1000$ ), again diluted in $1 \%$ BSA in TBS antibody solution, and then applied for $1 \mathrm{~h}$ at room temperature. Sections were then washed with TBS $2 \times 10 \mathrm{~min}$ or, if desired, incubated in TBS with DAPI (Roth) for $30 \mathrm{~min}$ before washing again $2 \times 10 \mathrm{~min}$. Slides were coverslipped $(24 \times 50 \mathrm{~mm}$ coverslips, Menzel Gläser) with Immu-Mount (Shandon Thermo Scientific) and sealed with clear nail polish once hardened.

\section{Nissl stain}

Sections were washed 3 times in PBS, then mounted onto Superfrost slides, and allowed to dry for 1-2 d. Slides were inserted into slide racks and passed through the following solutions: $95 \% \mathrm{EtOH}$ (ethanol from Roth; diluted as appropriate with $\left.\mathrm{ddH}_{2} \mathrm{O}\right) \times 15 \mathrm{~min}, 70 \% \mathrm{EtOH}, 50 \%$ $\mathrm{EtOH}, \mathrm{ddH}_{2} \mathrm{O}, 10 \mathrm{~min}$ Blue counterstain (TACS from Trevigen), $\mathrm{ddH}_{2} \mathrm{O}$, and then dehydrated through $50 \%, 70 \%, 95 \%$, and $100 \% \mathrm{EtOH}$ before clearing in Roti Histol (Roth), coverslipped using mounting medium (Entellan), and left to dry for $24 \mathrm{~h}$ under a fume hood.

\section{Confocal z stack image acquisition and processing}

Images were acquired on a spinning disc confocal microscope (Carl Zeiss Axio Observer.Z1 with Andor spinning disc and cobolt, omricron, i-beam laser) using a $40 \times(1.3 \mathrm{NA})$ and $100 \times(1.4 \mathrm{NA})$ Plan-Apochromat oil objective and an iXon ultra (Andor) camera controlled by iQ software (Andor). Sections for $\mathrm{GABA}_{\mathrm{A}} \alpha 6$ analysis (see Fig. 7) were imaged with a Nikon Spinning Disk Confocal CSU-X using a 100× (1.45 NA) Plan Apo oil objective and an EMCCD camera with Andor Revolution SD System (Andor).

\section{Tile scan overview images}

An upright microscope (Olympus, BX61) was used to image fluorescently stained cerebellar sections. A CCD color camera was used with a $10 \times(0.3 \mathrm{NA})$ or $2 \times(0.06 \mathrm{NA})$ lens for overview pictures. CellSens software (Olympus) stitched multiple images together to give an overview of the whole cerebellar region.

\section{Image analysis}

For image processing and analysis, ImageJ/FIJI software was used (Schindelin et al., 2012). For analysis of $P c l o^{w t / w t}$ and $P c l o^{g t / g t}$, tissue sections were selected from the vermis, and approximately every 10th slide laterally (each slide containing 2 sections) was analyzed. The best-quality section per slide was imaged. For layer thickness, all lobes were measured; and for closer analysis of MF size, climbing fiber (CF) coverage, and GC density, images were taken from lobes I, III, V, VII, and IX where possible.

Layer "thickness" was calculated per lobe by dividing the area of the layer by the inner length of the layer for both GC layer (GCL) and molecular layer (ML). For PC density, the number of PCs per lobe was counted and divided by the length of the PC layer for that lobe. Data points represent "thickness" values from individual lobes.

GC density was calculated from $100 \times$ magnification image stacks stained with DAPI. GCs were counted according to Mayhew and Gundersen (1996) using the unbiased optical dissector method, where 3D tissue can be quantified by dividing it into multiple 2D sections (focus planes of a confocal $Z$ stack). Each section is then assessed, and only the cells that exist in their entirety within the tissue slice, not cut at the edges or at the top or bottom of the slice can be counted. This method removes confounding factors, such as shape and orientation of the cells from the analysis. The experimenter was blinded to the genotype of each image. Data points represent each image.

MF cluster size was measured using a script within ImageJ to analyze particles stained with VGluT1 and/or VGluT2. The area of each puncta was measured; data points represent average cluster size of each animal.

$\mathrm{CF}$ innervation was assessed using VGluT2 and calbindin staining. The ML area was defined in the calbindin channel, consisting of calbindinpositive PC dendrites, and the VGluT2 channel was selected and pasted into a new ImageJ file. The same script as for MF analysis was run to calculate the total area stained with VGluT2, divided by the area of the $\mathrm{ML}$ and $\times 100$ to give percentage coverage. Data presented represent percentage per image.

For $\mathrm{GABA}_{\mathrm{a}} \alpha 6$ subunit expression, average signal intensity was measured for the whole FOV in the GABA $\alpha 6$ antibody channel and then normalized to the MAP2 channel for each image.

Due to the severity of the genotype, it was not possible to blind the experimenter when acquiring images and for some of the analysis. However, analysis of GC density was conducted blind; MF and CF analyses were conducted using an ImageJ script (therefore immune to experimenter bias).

\section{Electron microscopy (EM)}

$P c l o^{w t / w t}$ and $P c l o^{g t / g t}$ rats were anesthetized deeply with $33 \mathrm{mg} / \mathrm{ml}$ ketamine (Inresa Arzneimittel), $830 \mu \mathrm{g} / \mathrm{ml}$ Xylavet (cp-pharma), and perfused transcardially with $37^{\circ} \mathrm{C}$ physiological saline for 3-4 min followed by a $0.1 \mathrm{M}$ PB containing $4 \%$ PFA and $0.05 \%$ glutaraldehyde. Brains were stored in fixative overnight and subsequently sliced sagittally $(350 \mu \mathrm{m})$ on a vibratome. ROIs were cut into small pieces, postfixed in $1 \% \mathrm{OsO}_{4}$ and $0.1 \mathrm{M}$ sodium cacodylate, and en bloc stained in $1 \%$ uranyl acetate aqueous solution. Finally, samples were dehydrated and embedded in epoxy resin (Epon 812; EMS). Ultrathin sections were cut using an Ultracut ultramicrotome (UCT, Leica Microsystems) equipped with a diamond knife (Ultra 45, DiATOME) and collected on formvar-coated 200-mesh copper grids (EMS). Sections were imaged in an EM-900 Transmission Electron Microscope (Carl Zeiss) operated at $80 \mathrm{keV}$, and equipped with a $2 \mathrm{~K} \times 2 \mathrm{~K} C \mathrm{CD}$ camera. Data were analyzed blindly using the ImageJ software. However, due to the extreme phenotype at the EM level, it was not possible to be completely blind. Data points represent analysis from individual images obtained from 3 rats per genotype.

For histological and EM data, a normality test was performed (D'Agostino-Pearson Omnibus Normality Test). If successful, then a Student's $t$ test was used to compare $P c l o^{w t / w t}$ with $P c l^{g t / g t}$ rats, or alternatively a test for non-normal data (Mann-Whitney $U$ test) was used.

\section{Electrophysiology methods}

Cerebellar slices were prepared from 3- to-4-month-old Pclo ${ }^{w t / w t}$ and $P c l o^{g t / g t}$ rats of either sex. Animals were anesthetized with isoflurane 
(Baxter) followed by a rapid decapitation with a custom-built guillotine. The cerebellar vermis was quickly removed and mounted in a chamber filled with chilled extracellular solution. Parasagittal $300 \mu \mathrm{m}$ slices were cut using a VT1200 microtome (Leica Microsystems), transferred to an incubation chamber at $\sim 35^{\circ} \mathrm{C}$ for $30 \mathrm{~min}$, and subsequently stored at room temperature. ACSF was used for slice cutting, storage, and experiments. ACSF contained the following: $125 \mathrm{~mm} \mathrm{NaCl}, 25 \mathrm{~mm} \mathrm{NaHCO}_{3}$, $2.5 \mathrm{~mm} \mathrm{KCl}, 1.25 \mathrm{~mm} \mathrm{NaH}_{2} \mathrm{PO}_{4}, 1.1 \mathrm{~mm} \mathrm{CaCl}_{2}, 1 \mathrm{~mm} \mathrm{MgCl}_{2}, 3 \mathrm{~mm}$ glucose, and $17 \mathrm{~mm}$ sucrose ( $\sim 310 \mathrm{mOsm}, \mathrm{pH} 7.3$ when bubbled with carbogen, $5 \% \mathrm{O}_{2} / 95 \% \mathrm{CO}_{2}$ ). Patch pipettes were pulled from borosilicate glass (Science Products) using a DMZ Puller (Zeitz Instruments). Patch pipettes had open-tip resistances of $6-9 \mathrm{~m} \Omega$. The intracellular solution contained the following: $150 \mathrm{~mm} \mathrm{~K}$-gluconate, $10 \mathrm{~mm} \mathrm{NaCl}, 10$ mM K-HEPES, 3 mм Mg-ATP, and 0.3 mm Na-GTP (300-305 mOsm, $\mathrm{pH}$ adjusted to 7.3 with $\mathrm{KOH}$ ). In some of the experiments, the intracellular solution contained $10 \mu \mathrm{M}$ of the fluorescence dye Atto594. Experiments were performed at $35^{\circ} \mathrm{C}-37^{\circ} \mathrm{C}$, and slices were continuously superfused with ACSF containing $20 \mu \mathrm{M}$ SR95531 and $40 \mu \mathrm{M}$ D-APV to block Golgi-cell inhibition and NMDARs, respectively. Atto594 was obtained from Atto-Tec; all other chemicals were purchased from Sigma Millipore.

\section{Current-clamp recordings}

To determine the input resistance, subthreshold current pulses were applied from -20 to $20 \mathrm{pA}$ in $2 \mathrm{pA}$ steps. Action potentials (APs) were evoked in current-clamp mode by current pulses (amplitude 20-400 pA, duration $300 \mathrm{~ms}$ ). The access resistance was $24.9 \pm 1$ and $23 \pm 1 \mathrm{M} \Omega$ for $\mathrm{Pclo}^{w t / w t}$ and $\mathrm{Pclo}^{g t / g t}$ rats, respectively. Patch-clamp recordings were made using an EPC10/2 USB amplifier (HEKA Elektronik). Data were sampled at $200 \mathrm{kHz}$. Measurements were corrected for a liquid junction potential of $16 \mathrm{mV}$.

The current-clamp data were analyzed using custom-made procedures in Igor Pro software (WaveMetrics) as described previously (Eshra et al., 2019). In short, properties of APs of GCs were determined from the injected currents that elicited the largest number of APs. The AP threshold was defined as the membrane voltage at which the first derivative exceeded $100 \mathrm{~V} \mathrm{~s}^{-1}$, the minimal AP peak was set as $-20 \mathrm{mV}$, and the minimal amplitude to $20 \mathrm{mV}$. All APs with a half-width $<50 \mu$ s and $>500 \mu$ s were excluded. AP frequency and AP half-width were calculated from the first three APs. Membrane capacitance, resting membrane potential, and series resistance were read from the amplifier software (HEKA Elektronik) after achieving the whole-cell configuration. Input resistance was analyzed by plotting the steady-state voltage elicited by the subthreshold current injections against the injected current, and a spline interpolation was performed to obtain the slope at the holding membrane potential ( $0 \mathrm{pA}$ current injection).

\section{EPSCs}

To measure evoked EPSCs, GCs were held at a holding potential of $-80 \mathrm{mV}$ and $\mathrm{MF}$ axons were stimulated at $1 \mathrm{~Hz}$. The stimulation voltage ranged between 16 and $40 \mathrm{~V}$ for control and $P c l o^{g t / g t}$ animals. For spontaneous EPSCs, GCs were held at $-80 \mathrm{mV}$ for $\sim 3 \mathrm{~min}$. Single events were detected using the Igor Pro extension NeuroMatic (Rothman and Silver, 2018) tool for event detection. TTX was not used to prevent spontaneous MF APs, as the somata of MF axons are located outside of the cerebellum. As no hillock is present in the slice preparation, no spontaneous APs can be initiated. Due to obvious differences in cerebellar size, it was not possible to blind the experimenter during slice recordings.

EPSCs were analyzed with the Igor Pro software. The amplitude and the kinetics were determined from the average of 25 individual single EPSCs. To obtain the decay kinetics, single EPSCs were fitted with one or two exponentials. The weighted time constant was calculated as follows:

$$
\tau w=\frac{\text { Aslow } \tau \text { slow }+ \text { Afast } \tau \text { fast }}{\text { Aslow }+ \text { Afast }}
$$

\section{Behavioral assessment}

Rotarod task. The apparatus (IITC Life Science) consisted of 5 semienclosed lanes and an elevated metal rod $(9.525 \mathrm{~cm}$ diameter, $29.21 \mathrm{~cm}$ elevation) with a fine textured finish to enhance grip. For each trial, all rats were placed on the unmoving rod, allowed to stabilize their posture, and then rod rotation was initiated. Test parameters were as follows: rotation direction, toward investigator to encourage rats to face away while walking; start speed, $4 \mathrm{rpm}$; top speed, $44 \mathrm{rpm}$; acceleration rate, 0.2 $\mathrm{rpm} / \mathrm{s}$ (200 s from start to top speed); maximum test duration, $300 \mathrm{~s}$. Each rat's trial ended when it fell from the rod, triggering the falldetection sensor. Data were automatically recorded to a computer. Rats underwent 4 trials/d, with an intertrial interval of at least 10 min for 4 consecutive days.

Ladder rung task. Ladder rung tests were performed on cohorts of $P c l o{ }^{w t / w t}, P c l o{ }^{w t / g t}$, and $P c l o^{g t / g t}$ rats by methods previously described (Metz and Whishaw, 2009). Rats were trained to cross a Plexiglas tunnel $\sim 1 \mathrm{~m}$ long with metal rods provided at regular intervals as steps. Each step that the rat took was scored on the basis of paw placement on a 7 category scale, with 0 being paw totally missing the rung and 6 being correct paw placement. The average score per pair of limbs was used to quantify step score. During the trial, the error per step was also quantified by number of low scoring steps $(0-2)$ divided by the total number of steps that the rat took during the test with each pair of limbs (Student's $t$ test). Data were derived from three trials with $3 \mathrm{~cm}$ rung separation conducted on the same test day with at least a 10 min intertrial interval, and group means were compared using one-way ANOVA and Bonferroni post hoc tests.

Grip test. To assess grip strength, rats were allowed to cling on to a support by forelimbs or hindlimbs and pulled (Curzon et al., 2009). The support is attached to a transducer that can measure the pull force being applied on the rat by the tester. During each trial, force that was necessary to be applied by the tester to release the grip of the rat was recorded. Three trials per limb pair were done and the means compared by Student's $t$ test. To assess grip strength, the rat's torso was supported ventrally while both forelimbs were allowed to grasp a metal support bar; then the rat was pulled in a horizontal plane until the bar was released. Peak force was measured by a transducer attached to the support bar (San Diego Instruments). Nine trials per rat were conducted over $2 \mathrm{~d}$ (intertrial interval at least $5 \mathrm{~min}$ ), and group means were compared using one-way ANOVA and Bonferroni post hoc tests.

Open field test. The open field test (Curzon et al., 2009) was performed for each rat using a rectangular arena $(91.44 \mathrm{~cm} \times 60.96 \mathrm{~cm})$ divided into 9 equal sectors ( 1 center, 8 perimeter). At the start of the experiment, the rat was placed in the center sector and allowed to explore for $10 \mathrm{~min}$. The test was recorded by digital video for later analysis. One test was conducted per rat. The behaviors counted were crossing, rearing, and grooming. Each crossing event was counted when all four limbs of the test subject crossed the boundary of one sector into another. Rearing was defined as the number of times the subject stood up on its hind limbs. Grooming was defined as the number of times a rat licked or scratched itself while remaining stationary. Events that occurred in the center versus perimeter sectors were tallied separately, and group means were analyzed for each region by one-way ANOVA and Bonferroni post hoc tests.

During the above experiments, the experimenter was blinded to the genotype of the animals during data acquisition and analysis.

OptiMan (Operator Independent Motor-Analysis, PhenoSys) system.

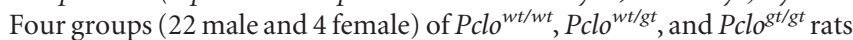
were used in this study. Rats carried subcutaneous radiofrequency identification (RFID) tags in ventral location. Animals were group-housed with $6-8$ rats per group. Initially, all rats were habituated to the OptiMan multicage environment with open sorter gates for voluntary exploration. Then, automated sorting was activated so that only one rat could enter the operant chamber at a time. During pull-task training, force thresholds and handle positions were adapted every day for each session to the current skill level reached by a rat. Each session had 20 trials, and two or three individual sessions were given per day with an intersession interval of 30-60 min. Within a session, the maximum time interval a rat was allowed to remain inactive between trials was 6-8 min. A session ended when such inactivity occurred. The OptiMan system consists of two interconnected group home cages (EU Type IV cage and 2000P) resting on an RFID sensor array plate that automatically tracks RFID tag movement within the cage, and thus the locomotor activity of individual rats, day and night. One of the home cages was connected via an electronic 

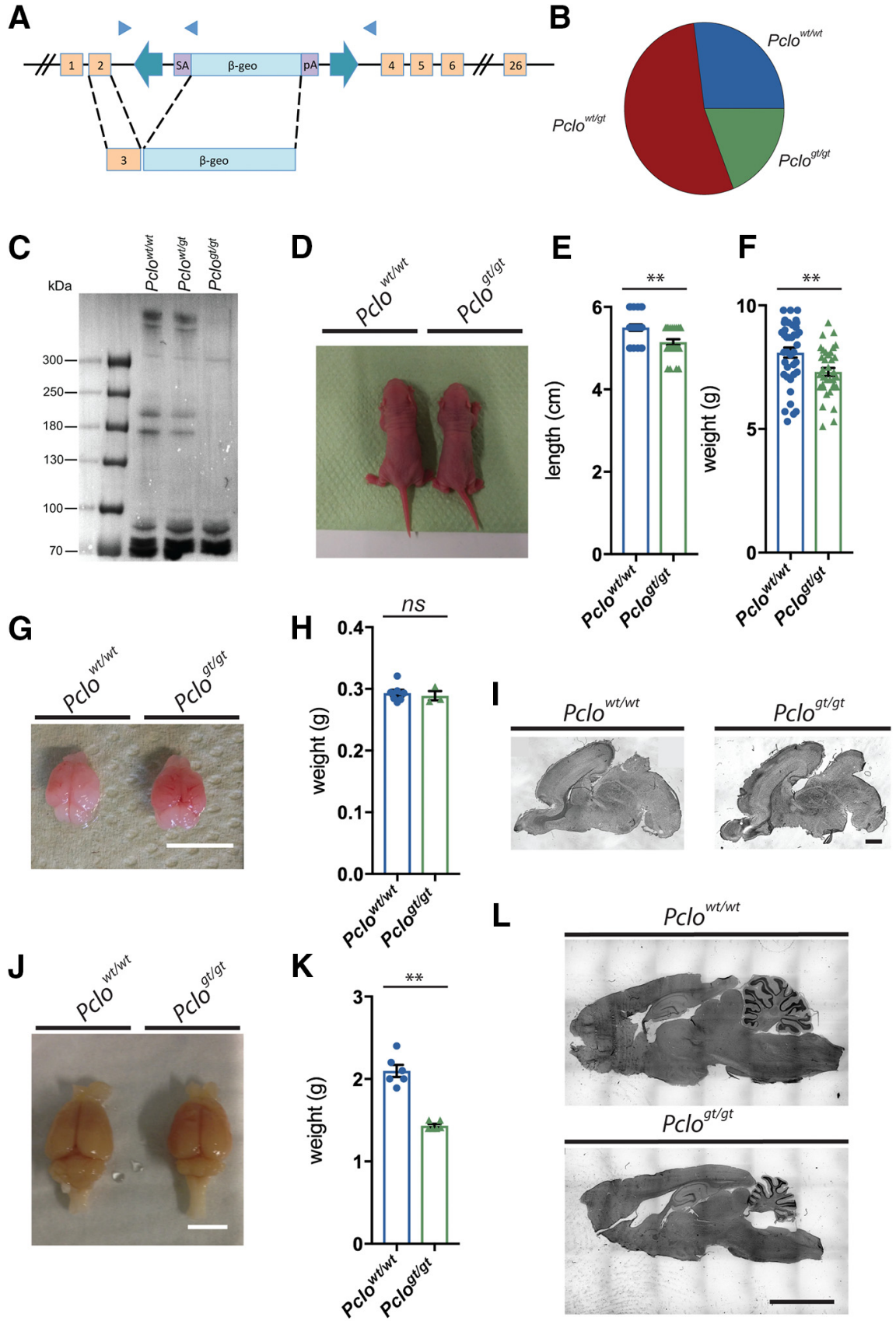

Figure 1. Generation of $P$ clot $0^{g t / t}$ mutant animals. A, Sleeping beauty transposon mutagenesis was used to generate gene trap (gt) piccolo KO rats. The transposon element was inserted into exon 3 of the piccolo genomic sequence and caused a stop in the reading frame. Adapted from Ackermann et al. (2019). B, Pairs of heterozygous $\left(P C / 0^{w t / g t}\right)$ males and females produced litters with Mendelian distribution. Pie chart demonstrates the birth rates of homozygous WT ( $P\left(10^{\mathrm{wt} / \mathrm{wt}}\right)$, homozygous gene trap mutation $\left(P \mathrm{cl} 0^{g t / g t}\right)$, and heterozygous $\left(P \mathrm{Cl} 0^{\mathrm{wt} / g t}\right.$ ) pups (data collected from 63 litters: $P$ clo ${ }^{\mathrm{wt} / \mathrm{wt}}=195 \mathrm{pups} ; P_{\mathrm{cl}} 0^{\mathrm{wt} / g t}=387 \mathrm{pups} ; P_{\mathrm{cl}} 0^{g t / g t}$ $=137$ pups). $C$, Western blot analysis of brain lysates prepared from postnatal day 2 (P2) animals to confirm the loss of full-length Piccolo protein from the brain. A band of the Piccolo-corresponding size of $560 \mathrm{kDa}$ is detectable in lysates prepared from $P$ clo ${ }^{\mathrm{wt} / \mathrm{wt}}$ and $P\left(10^{\mathrm{wt} / g t}\right.$ animals, as well as bands between 70 and $420 \mathrm{kDa}$, representing smaller Piccolo isoforms. Nearly all of these are absent in $P$ cl $0^{g t / g t}$ brain lysates (data are representative of three independent experiments), although smaller bands between 100 and $70 \mathrm{kDA}$ are still present in $P$ clo ${ }^{g t / g t}$ brain lysates. $\boldsymbol{D}$, Image of postnatal day 1 (P1) littermates. $\boldsymbol{E}$, Quantification of the body length of P0-P2 $P$ clo ${ }^{w t / w t}$ and $P$ clo $0^{g t / g t}$ pups $\left(P c l 0^{w t / w t}=5.5 \pm 0.077 \mathrm{~cm}, n=23 ; P\right.$ clot $0^{g t / g t}=5.15 \pm 0.070 \mathrm{~cm}, n=27 ; 6$ independent litters; unpaired $t$ test, $\left.t_{(48)}=3.394,{ }^{* *} p=0.0014\right)$. $\boldsymbol{F}$, Quantification of the body weight of P0-P2 $P$ clo ${ }^{w t / w t}$ and $P c l 0^{g t / g t} \operatorname{pups}\left(P c l 0^{w t / w t}=8.09 \pm 0.203 \mathrm{~g}, n=39 ; P c 10^{g t / g t}=7.31 \pm 0.166 \mathrm{~g}, n=35 ; 12\right.$ independent litters; unpaired $t$ test, $t_{(72)}=$ 2.937 , $\left.{ }^{* *} p=0.0044\right)$. G, Image of brains dissected from P1 $P$ Cllowt/wt and $P$ cllot/gt pups. $\boldsymbol{H}$, Quantification of the brain weight of $P 0-P 2 P c 10^{\mathrm{wt} / \mathrm{wt}}$ and $P$ cl $0^{\mathrm{gt} / \mathrm{gt}}$ pups $\left(P \mathrm{cl} 0^{\mathrm{wt} / \mathrm{wt}}=0.293 \pm 0.00533 \mathrm{~g}, n=7 ; P \mathrm{cl} 0^{\mathrm{gt} / \mathrm{gt}}=0.289 \pm 0.00758 \mathrm{~g}, n=3 ; 3\right.$ independent litters; unpaired $t$ test, $\left.t_{(8)}=0.423, p=0.683\right)$. II, Nissl-stained sagittal sections from P2 rat brains show no overt differences between $P$ clo $0^{g t / g t}$ and $P$ clowt/wt pups. Animals used in this experiment are as follows: wt 1; k01.J, Image of 4\% PFA-perfused brain from $P$ clo ${ }^{\mathrm{wt} / \mathrm{wt}}$ and $P \mathrm{Cl} 0^{g t / g t}$ animals at 3 months of age. $K$, Quantification of the brain weight showing that $P$ clo $0^{g t / g t}$ brains are significantly lighter than $P c / 0^{w t / w t}$ brains $\left(P C l 0^{w t / w t}=2.098 \pm 0.074 \mathrm{~g}, P c 10^{g t / g t}=1.435 \pm 0.021 \mathrm{~g} ; n=6\right.$, Mann-Whitney $\mathrm{U}$ guillotine gate to a second cage resting on a balance that automatically determined the body mass of a rat when inside this cage. Individual animals voluntarily and self-motivated decided to visit the balance cage. Subsequent to the balance cage, and again separated by an electronic guillotine gate, was a cage compartment containing a horizontal ladder $(1 \mathrm{~m})$ with electronically monitored dual rungs on the left and right side. From this ladder compartment, an animal entered a cage containing the isometric pull-task. From there back to the home cage, a rat passed over a force grid sensor array that sensed ground forces exerted by the paws.

Isometric pull task. Rats were trained to pull a handle attached to a stationary force transducer with a predetermined force threshold upon which they received a sugar pellet reward. Upon a rat's entry into the isometric pull task cage, a sugar pellet was delivered into the reward tray. A session started when the rat retrieved this first reward, which led to the automatic closing of the entry gate, and to the motorized slide-in appearance of the force handle to its predefined position. During each session, 5 different handle positions were presented to a rat. These positions varied from 11 $\mathrm{mm}$ inside the cage wall (easiest position) to 7 $\mathrm{mm}$ outside of the cage wall (most difficult position). Handle positions changed automatically during a session through a motorized slide. The difficulty level within each handle position increased stepwise by increasing the force threshold for pellet release. This started at $30 \mathrm{~g}$ pull-force and was increased to 40,50 , and finally $60 \mathrm{~g}$. A trial started when a pull-force of $5 \mathrm{~g}$ was sensed. From then on, the pull-force was sampled for a duration of $4 \mathrm{~s}$. If a rat reached the force threshold within a $2 \mathrm{~s}$ time interval, then a trial was successful and a reward delivered. The schedule then advanced to the next level of difficulty by either increasing force threshold or moving the handle one position further toward the outside. Thus, a rat needed a minimum of 20 trials to complete a session with 5 different handle positions and 4 different force thresholds at each position. If the threshold was not reached, then a trial was considered unsuccessful and the rat had to continue with its next trial with unchanged conditions. Experiments lasted for 15 experimental days with two or three daily sessions per individual.

Due to the automated setup, experimenter bias is greatly reduced. Nonetheless, the experimenter was also blinded to the genotype of the rats during training and test phases.

\section{Animal welfare}

All animals were treated and cared for in accordance with national and institutional guidelines:

\section{$\leftarrow$}

test, $\left.U=0,{ }^{* *} p=0.0022\right) . L$, Nissl-stained sagittal sections from 3-month-old rat brains reveal microcephaly in $P$ clogt/gt compared with $P c l 0^{w t / w t}$. Ventricles are larger, and cerebellum, pons, cerebrum, and subcortical regions are smaller. Animals used in this experiment are as follows: wt11; ko13. Scale bars: $G, J, 1 \mathrm{~cm} ; I, 1 \mathrm{~mm} ; L, 0.5 \mathrm{~cm}$. Error bars indicate SEM. 
Generation of mutant Piccolo rat strains and behavioral experiments: Rat protocols were approved by the Institutional Animal Care and Use Committee at the University of Texas Southwestern Medical Center in Dallas, as certified by the Association for Assessment and Accreditation of Laboratory Animal Care International (permit number: NIH OLAW Assurance \#D16-00296).

Western blotting, immunohistochemistry, $\mathrm{EM}$, and behavioral experiments with Optiman setup: Animals were treated in accordance with the German Protection of Animals Act (TierSchG $\$ 4$ Abs. 3 ); all procedures for experiments involving animals were approved by the animal welfare committee of Charité Medical University and the Berlin state government (permit number: T0036/14).

Electrophysiology: Animals were treated in accordance with the German Protection of Animals Act (TierSchG $\$ 4$ Abs. 3) and with the guidelines for the welfare of experimental animals issued by the European Communities Council Directive of November 24, 1986 (86/ 609/EEC). The local authorities approved the experiments (Landesdirektion Leipzig; permit number: T24/18)

Sixty-nine animals were used for histology, EM, electrophysiology, and behavior experiments. These animals have been numbered wt1-34 and ko1-35; the animal IDs for each experiment can be found in the figure legends.

\section{Experimental design and statistical} analysis

GraphPad Prism (RRID:SCR_002798) was used to analyze and represent data. Statistical design, sample sizes, and tests for each experiment can be found in the figure legends.

\section{Results}

\section{Brain morphology is changed in Piccolo KO brains}

The recent analysis of 2 boys with $\mathrm{PCH} 3$ identified a non-sense mutation in the coding region of the human Pclo gene (chr7: $82579280 \mathrm{G}>\mathrm{A}$ ), predicted to eliminate the C-terminal third of the longest Piccolo isoforms (Ahmed et al., 2015) and likely their expression. These individuals have profound cognitive and motor impairment as well as atrophy of the cerebrum, cerebellum, and pons (Ahmed et al., 2015). A fundamental question is whether Piccolo LOF is causal for this disorder. To explore this possibility, we have characterized a recently generated line of rats $\left(P c l o^{g t / g t}\right)$ wherein transposon mutagenesis was used to disrupt the Piccolo gene, with 25 exons, via an insertion into exon 3 (Fig. 1A). This insertion is predicted to cause a frame shift in the reading frame and thus disrupt the expression of full-length Piccolo $(560 \mathrm{kDa})$ and most of its alternatively spliced lower molecular weight isoforms $(70-350 \mathrm{kDa})$ (Fig. 1A). Schematic is adapted from Ackermann et al. (Ackermann et al., 2019; Medrano et al., 2019). Just after birth (postnatal day 0-2, P0-P2), Piccolo pups were found born in normal Mendelian numbers (Fig. 1B). Western blot analysis of brain lysates from P0-P2 pups demonstrates the loss of nearly all Piccolo isoforms in homozygous $\mathrm{KO}$ animals (Fig. 1C). To assess whether Piccolo LOF adversely affects brain development, we performed an anatomical characterization of $P c l o^{w t / w t}$ and $P c l o^{g t / g t}$ animals; $P c l o^{g t / g t}$ pups were smaller and weighed significantly less than their Pclo ${ }^{w t / w t}$ littermates (mean length, weight: $\left.P c l o^{w t / w t}=5.50 \mathrm{~cm}, 8.09 \mathrm{~g} ; P_{c l o}^{g t / g t}=5.15 \mathrm{~cm}, 7.31 \mathrm{~g}\right)($ Fig.
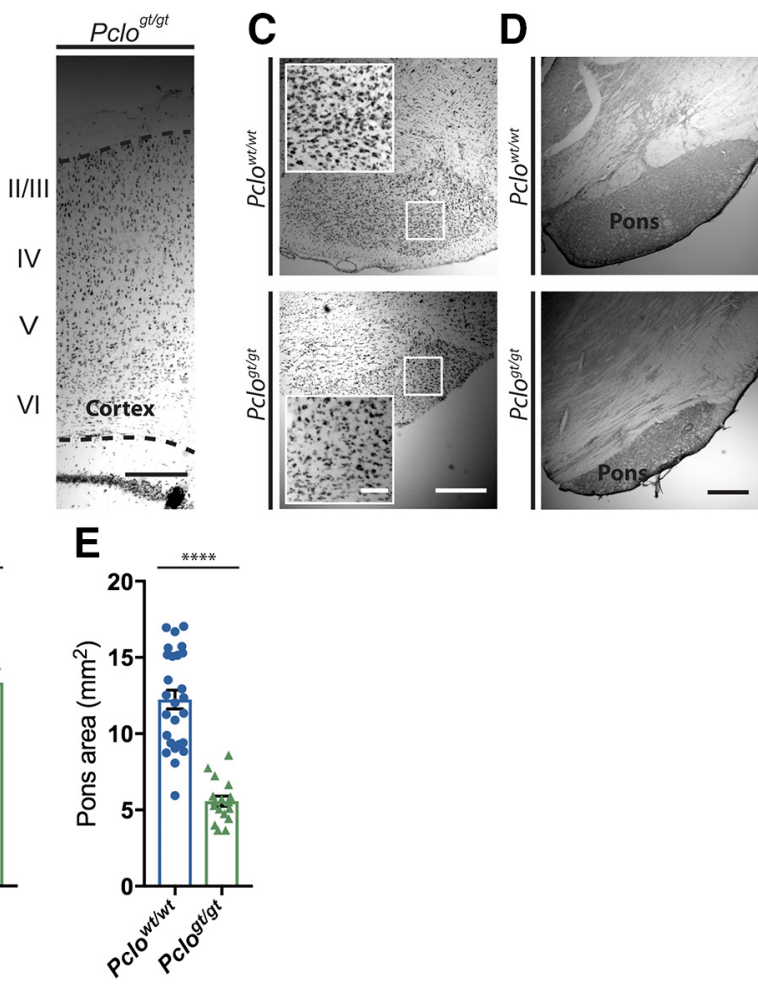

Figure 2. PClo ${ }^{g t / g t}$ rats show cortical thinning and a smaller pons area compared with $P$ Clo $0^{w t / w t}$ littermates. $A, C, D$, Nissl-stained

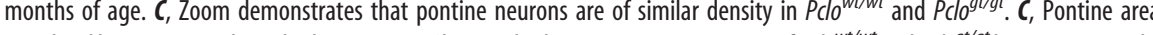
$A, C, 200 \mu \mathrm{m} ; \boldsymbol{B}, \boldsymbol{C}, 500 \mu \mathrm{m} ; \boldsymbol{B}(\mathrm{zoom}), 100 \mu \mathrm{m}$. Error bars indicate SEM. Animals used in this experiment are as follows: wt3, wt7-wt10; k03, k06, k08, k010.

$1 D-F)$. However, brain weights were not significantly altered between $\mathrm{Pclo}^{g t / g t}$ and $\mathrm{Pclo}{ }^{w t / w t}$ littermates at P0-P2 and display similar brain morphology (Fig. $1 G-I$ ), suggesting that changes in brain size develop postnatally. The weight of $P c l o^{g t / g t}$ brains was significantly reduced by $31.6 \%$ in 3 -month-old adult rats compared with $P c l o^{w t / w t}$ brains (Fig. $1 \mathrm{~J}, \mathrm{~K}$ ). To assess whether this was associated with an overall loss in brain volume or due to reductions in specific brain regions, serial sagittal and coronal sections from 3-month-old animals were collected and stained with Nissl to visualize brain morphology (Fig. $1 L$ ). Qualitative analysis revealed that thalamic, cerebellar, and brainstem regions are dramatically reduced in size in 3-month-old $\mathrm{Pclo}^{g t / g t}$ animals. Some thinning of the cerebral cortex can also be observed; however, no obvious changes in the hippocampus are detectable. Furthermore, ventricles (V1, V2, V3, and V4) in $P c l o^{g t / g t}$ brains were all notably larger than in $P c l o^{w t / w t}$ littermates (Fig. $1 \mathrm{~L}$ ).

Intriguingly, the observed morphological changes in brains of 3-month-old $P c l o^{g t / g t}$ animals are remarkably similar to those reported for patients with $\mathrm{PCH} 3$, who exhibit microcephaly, a reduced size of the cerebrum, cerebellum, and pons as well as larger ventricles (Maricich et al., 2011). As hypoplasia of the pons and cerebellum as well as a reduced thickness of the cortex are the most dramatic features of $\mathrm{PCH} 3$ patients, we examined in more detail changes occurring in these regions of $\mathrm{Pclo}^{g t / g t}$ brains. Here, we found that the thickness of the cortex is significantly reduced by $9.2 \%$ in brains lacking Piccolo (Fig. $2 A, B$ ). The area of the 
A

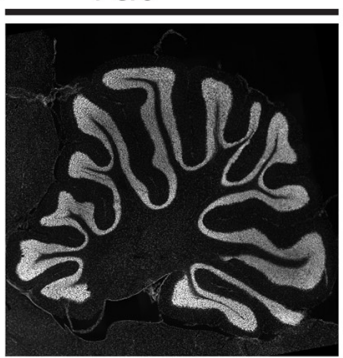

B

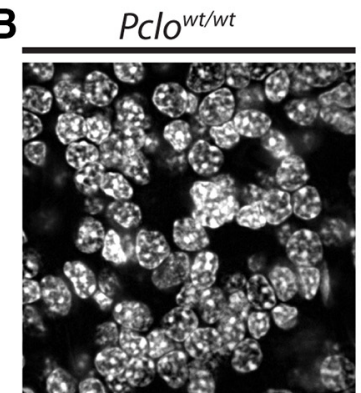

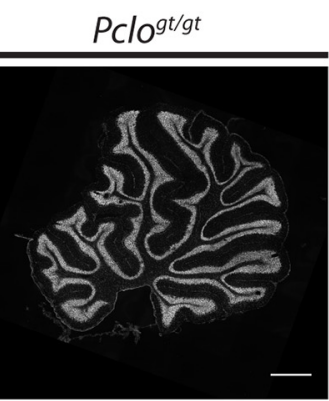

Pclogt/gt

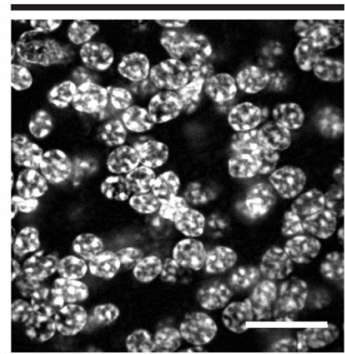

C

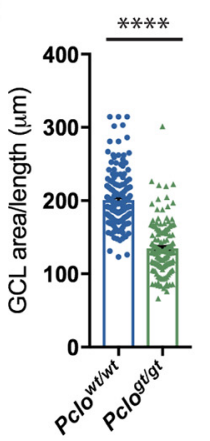

D

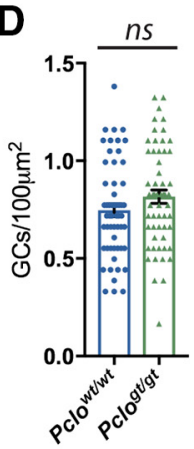

E

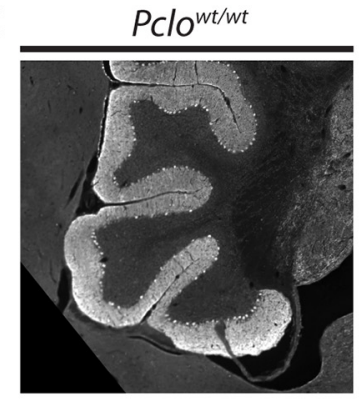

$F$

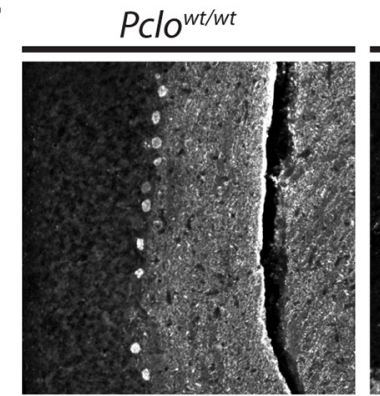

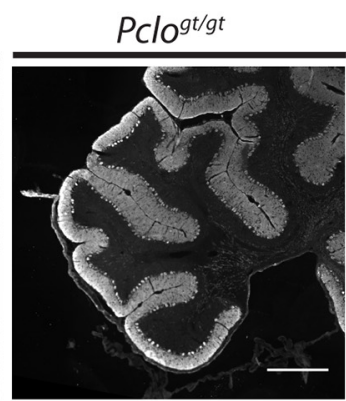

Pclogt/gt

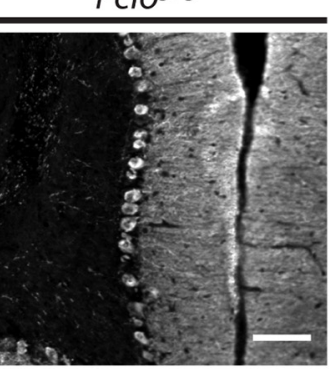

G $\quad * * * * \quad \mathbf{H}$

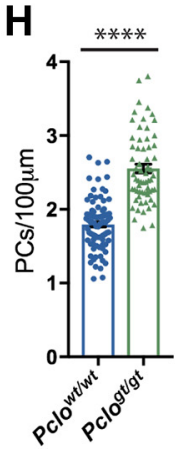

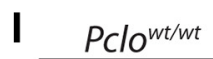

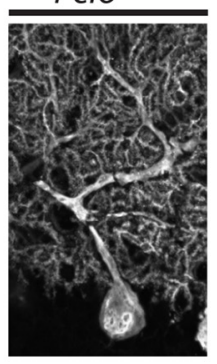

Pclogt/gt

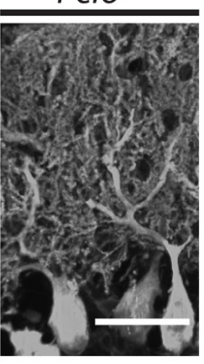

Figure 3. Morphometric comparison of $P$ Cl $10^{g t / g t}$ and $P$ Clo $0^{w t / w t}$ cerebella. $A-D$, Images of sagittal sections of $P$ Clo $0^{w t / w t}$ and $P\left(10^{g t / g t}\right.$ cerebella at 3 months of age. The densely packed GCL is visualized by DAPI staining. $\boldsymbol{B}$, Higher-magnification images of $A$, demonstrating GC density in the GCL. C, Quantification of the GCL thickness (PClo $0^{\text {wt } / w t}=200.8 \pm 2.932 \mu \mathrm{m}, n=160$ lobes; $P$ clogt/gt $=$ $134.8 \pm 2.859 \mu \mathrm{m}, n=148$ lobes; $n=4$ independent experiments; Mann-Whitney $U$ test, $U=1953$, $\left.{ }^{* * * *} p<0.0001\right)$. Animals used in this experiment are as follows: wt4, wt6-wt8; k04-k07.

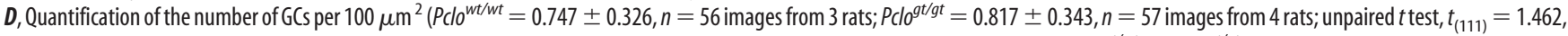
$p=0.147$ ). Animals used in this experiment are as follows: wt7, wt10, wt11; k06, k07, k09, k010. E-H, Images of sagittal sections of $P$ clowt/wt and $P$ clo ${ }^{\text {gt/gt }}$ cerebella at 3 months of age. PCs stained with antibodies against calbindin determine the ML (lobes I-III shown). $\boldsymbol{F}$, Higher-magnification images of $\boldsymbol{E}$. Note the closer packing of PCs in $P$ clo $0^{\text {gt } / g t}$ compared with $P$ cllowt/wt. $\mathbf{G}$, Quantification of the ML thickness $\left(P c l 0^{w t / w t}=187.2 \pm 2.719 \mu \mathrm{m}, n=148\right.$ lobes; $P$ clo ${ }^{g t / 9 t}=127.2 \pm 2.378 \mu \mathrm{m}, n=125$ lobes $; n=4$ independent experiments; Mann-Whitney $U$ test, $U=1368, * * * * 0$ 0.0001). Animals used in this experiment are as follows: wt7, wt10, wt11; k06, k07, k09, ko10. $\boldsymbol{H}$, Quantification of the number of $\mathrm{PCs}$ per $100 \mu \mathrm{m}$ length of $\mathrm{PC}$ layer ( $P$ clowt $/ \mathrm{wt}=1.797 \pm 0.036$; $n=89$ lobes; $P\left(l_{0}{ }^{g t / g t}=2.554 \pm 0.058 ; n=65\right.$ lobes $; n=3$ independent experiments; unpaired $t$ test, $\left.t_{(152)}=11.62,{ }^{* * * *} p<0.0001\right)$. Animals used in this experiment are as follows: wt4, wt7, wt8; k04, k06, k07. I, Images of sagittal sections stained with antibodies against calbindin showing that PCs migrate correctly to their position in the ML and are correctly oriented. Scale bars: $A$, 1 $\mathrm{cm} ; \boldsymbol{B}, 20 \mu \mathrm{m} ; \boldsymbol{I}, 50 \mu \mathrm{m} ; \boldsymbol{F}, 200 \mu \mathrm{m}$. Error bars indicate SEM. Data points represent images taken from lobes I, III, V, VIII, and IX; 4 sections per animal $(\boldsymbol{B}, \boldsymbol{D})$. ns, not significant.

pons is dramatically reduced in $P c l o^{g t / g t}$ brain slices stained with Nissl (Fig. 2C). As the density of neurons was not changed (Fig. $2 C$, zoom), these data indicate a loss of the total number of neurons within the pons. This conclusion is supported by data from brainstem sections stained with antibodies against the SV protein VGluT1, prominently expressed in pons neurons, which reveals a $46.63 \%$ smaller area occupied by these neurons (Fig. 2D,E).

Analysis of sagittal sections through the cerebellum of adult rats reveals that, while the cerebellum is smaller in $P c l{ }^{g t / g t}$ animals, the overall anatomy is not altered (Fig. 3A). For example, there are no remarkable defects in foliation of $\mathrm{Pclo}^{g t / g t}$ animals, with all lobes present and appearing to be formed normally at the vermis (Fig. $3 A, E$ ). Our analysis of the GCL, using DAPI to stain GC nuclei, reveals that this layer is significantly reduced in size in $P c l o^{g t / g t}$ compared with $P c l o^{w t / w t}$ controls (mean GCL size: Pclo ${ }^{w t / w t}$ $\left.=200.8 \mu \mathrm{m} ; P_{c l o}{ }^{g t / g t}=134.8 \mu \mathrm{m}\right)($ Fig. $3 A, C)$. However, this decrease was not associated with a proportional increase in GC packing density, as the number of GCs per GCL area is unchanged (Fig. $3 B, D$ ). These data indicate an overall loss of GCs in $P c l o^{\text {gt/gt }}$ cerebella. Conceptually, this is predicted to reduce the total number of GC PFs innervating PC dendrites in the ML, a situation that could lead to a thinner ML and perhaps an altered packing density of PCs.

Sections immunostained with antibodies against calbindin, which specifically labels PCs, reveals that the organization of the $\mathrm{ML}$ and the PC layer appear to be intact and that the dendritic arbors of PCs are correctly oriented (Fig. 3E,F,I). However, quantifying the average area per unit length of the ML reveals a $32.1 \%$ reduction in the size of this layer in $P c l^{g t / g t}$ brains (Fig. $3 G)$. Furthermore, cells in the PC layer appear overcrowded in $P c l o^{g t / g t}$ cerebella (Fig. $3 E, F, I$ ), a conclusion supported by data showing an increased packing density of PCs in Pclo ${ }^{g t / g t}$ brains $\left(\right.$ mean PCs per $\left.100 \mu \mathrm{m}: P c l o^{w t / w t}=1.797 ; P c l o^{g t / g t}=2.554\right)($ Fig. $3 H)$. 
Together, these data indicate a reduced number of GCs and a higher packing density of PCs in Pclo ${ }^{g t / g t}$ cerebella compared with $P c l o o^{w t / w t}$ controls.

\section{Loss of Piccolo alters CF and MF innervation in the cerebellum}

Anatomically, the cerebellum receives its major excitatory afferents in the form of CFs and MFs that project from the inferior olive or the brainstem/spinal cord, respectively (Leto et al., 2016). Both form glutamatergic synapses but terminate in different layers of the cerebellum (Apps and Garwicz, 2005). CFs form excitatory synapses onto the proximal branches of PC dendritic arbors, modulating the dynamic firing properties of PCs and motor learning (Hashimoto and Kano, 1998). In contrast, MFs terminate on the dendrites of GCs, which then provide direct excitatory input to PCs via their PF axons. Both also extend collaterals to the deep cerebellar nuclei before projecting into the cerebellar cortex (Shinoda et al., 1992).

In previous studies, we observed that Piccolo was present in the boutons of each of these excitatory synapses (Cases-Langhoff et al., 1996). This was confirmed by immunostaining cerebellar sections of $P c l o^{w t / w t}$ and $\mathrm{Pclo}^{g t / g t}$ with antibodies against Piccolo and the SV protein VGluT1 (Fig. $4 A, B)$. This revealed that Piccolo immunoreactivity was indeed present at each of these synaptic types and that this immunoreactivity was lost in cerebella from $\mathrm{Pclo}^{g t / g t}$ animals. To explore whether deficiencies in either could contribute to the anatomical and functional changes in the cerebellum, we initially analyzed potential differences in the CF input into the ML. Synaptic input from CFs onto PC dendrites, immunopositive for calbindin, was visualized with antibodies against VGluT2 (Miyazaki et al., 2003). Here our analysis of sagittal sections revealed the presence of a large number of VGluT2-positive puncta decorating calbindin-positive dendrites in $P c l o^{g t / g t}$ and $P c l o{ }^{w t / w t}$ sections (Fig. 4C,D). These data indicate that the loss of Piccolo does not affect the ability of CFs to project into the ML and form synapses with PC soma and dendrites. Qualitatively, VGluT2-positive puncta in $\mathrm{Pclo}^{w t / w t}$ and $\mathrm{Pclo}^{g t / g t}$ sections were of similar size and beautifully decorated both primary and tertiary PC dendrites, although the total number of puncta appeared more numerous in $\mathrm{Pclo}^{g t / g t}$ sections. Quantification of the total area of VGluT2 per ML supports this impression by demonstrating a $24.3 \%$ increase in VGluT2 coverage of the ML (Fig. 4D). We compared in a qualitative manner the distribution of the synaptic inputs from GCs onto PCs, the PF axons. The latter synapses were identified with antibodies against VGluT1 (Miyazaki et al., 2003). Although the ML is thinner in Pclogt/gt cerebella (Fig. 3A), we observed no overt changes in the intensity or distribution of VGluT1-positive puncta throughout the ML of Pclogt/gt animals compared with Pclo ${ }^{w t / w t}$ controls (Fig. 4A). This implies that PF
B
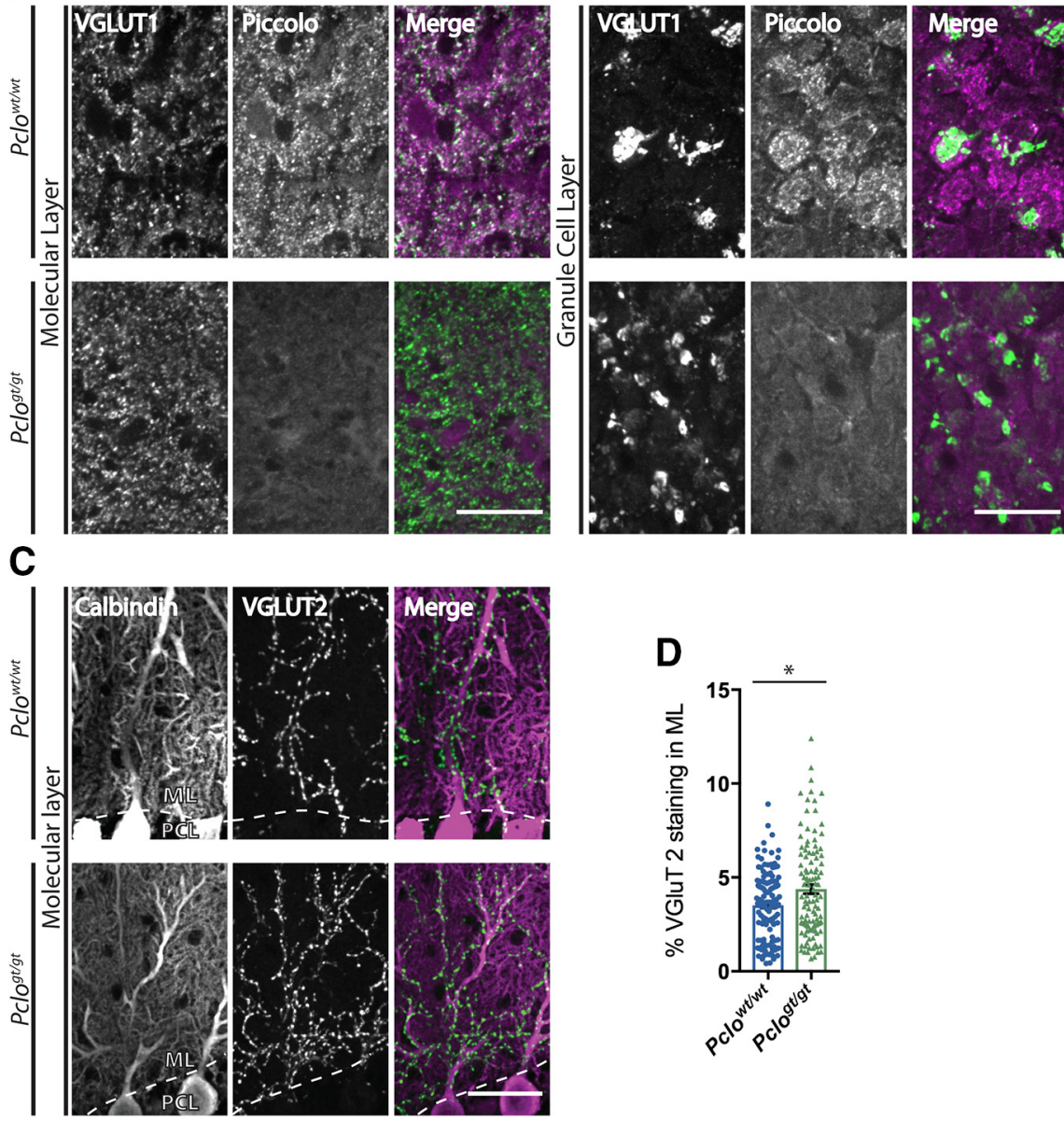

Figure 4. Aberrant CF innervation of $P C \sin P c l 0^{g t / g t}$ rats compared with $P\left(10^{w t / w t} . A, B\right.$, Images of sagittal sections of $P$ clo ${ }^{w t / w t}$ and $P$ clo $0^{g t / g t}$ cerebella at 3 months of age stained with antibodies against VGluT1 and Piccolo demonstrate the loss of Piccolo in the

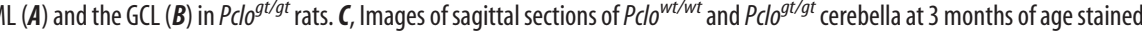
compared with $P$ Clo ${ }^{w t / w t}$ controls $(\boldsymbol{C}, \boldsymbol{D})$. When Piccolo is absent, PF synapses in the ML, immunopositive for VGluT1, do not appear overtly different between $P c 10^{w t / w t}$ and $P$ clo ${ }^{g t / g t}(\boldsymbol{A})$. However, MF and CF synapses are altered (B-D; see Fig. 5). D, images; $P$ clo ${ }^{g t / g t}=4.377 \pm 0.241, n=112$ images; $n=3$ independent experiments; Mann-Whitney $U$ test, $U=5987,{ }^{*} p=$ 0.0278). Animals used in this experiment are as follows: wt7, wt8, wt10, wt11; k06, k07, k09, k010. Scale bars, $20 \mu \mathrm{m}$. Error bars indicate SEM. Data points represent images taken from lobes I, III, V, VII, and IX; 4 sections per animal.

axons project normally and form a robust number of synapses with PCs. Given that fewer GCs are formed in Pclogt/gt cerebella (Fig. $3 A, C$ ), we postulate that the thinner ML is most likely due to fewer PFs and less total synaptic input on PC dendrites.

GCs are known to receive their excitatory MF input from afferent axons arising from a number of distinct nuclei in the brainstem, including the pontine nuclei (Sillitoe et al., 2012). These collaterals form large glomerular structures with multiple AZs, forming a rosette of synapses with claws from dendrites of multiple GCs (Jakab and Hámori, 1988; Xu-Friedman and Regehr, 2003). Given the smaller size of the pons, we thus explored whether the boutons from the remaining cells properly reached the cerebellum and formed robust MF terminals. As afferent fibers from the pons primarily innervate cerebellar lobes VI to IX, we examined sagittal sections stained with antibodies against the somatodendritic marker MAP2 and VGluT2 in lobe VII. In Pclo ${ }^{w t / w t}$ sections, multiple large VGluT2-positive puncta are seen packed tightly together within a dense meshwork of MAP2-positive dendrites projecting from a ring of GCs. These puncta represent 
A
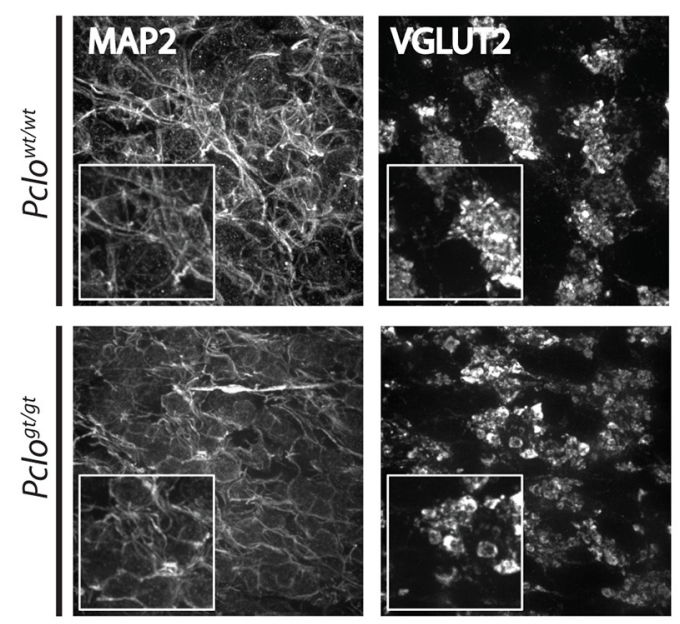

B

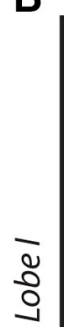
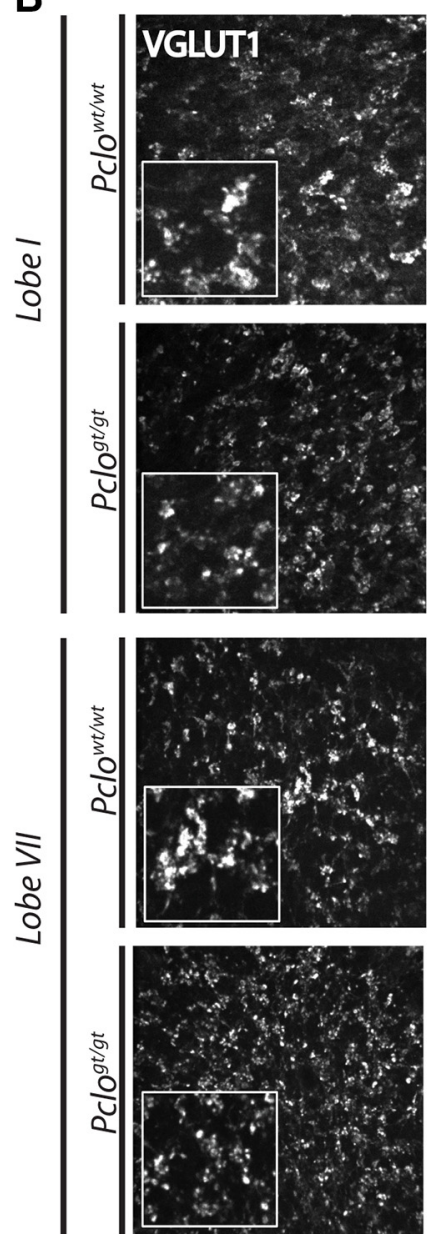
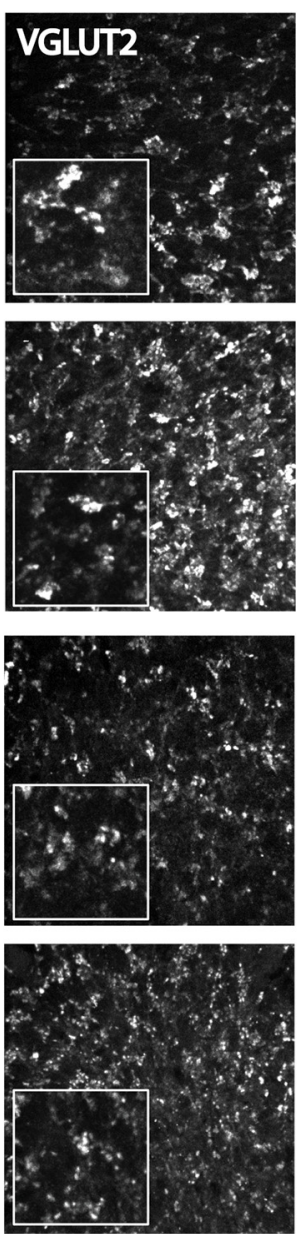
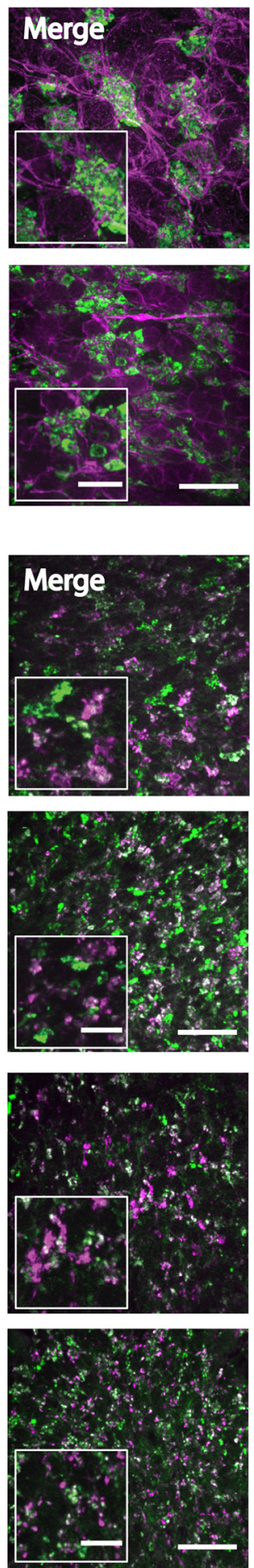

C

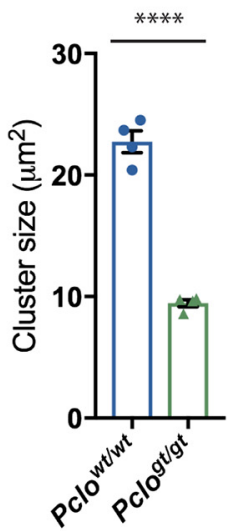

\section{D}

VGluT1/2 cluster size distribution smaller clusters: $5-50 \mu \mathrm{m}^{2}$
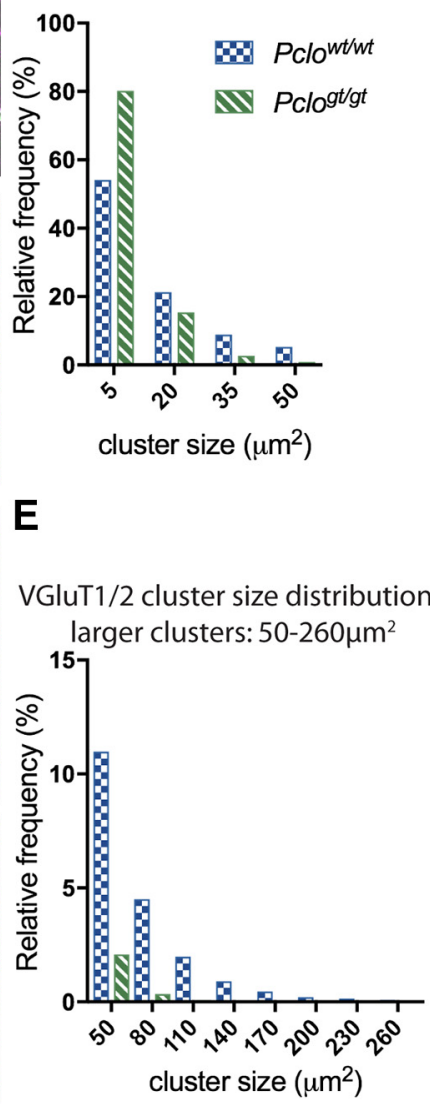

Figure 5. Cerebella from $P$ cl $0^{g t / g t}$ animals have smaller MF rosettes. $A, B$, Images of sagittal sections of $P\left(10^{w t / w t}\right.$ and $P\left(10^{g t / g t}\right.$ cerebella at 3 months of age stained with antibodies against VGluT2, which is highly expressed at MF boutons, and the somatodendritic marker MAP2 (A) or VGluT2 and VGluT1 (B). $\boldsymbol{A}$, GCs extend their dendrites into MF boutons in the $P$ clo ${ }^{\text {wt }} / w t$ condition. However, in $P C 10^{g t / g t}$, while GC dendrites are still juxtaposed to VGluT2-positive boutons, the boutons are smaller and therefore the arrangement is less organized. $\boldsymbol{B}$, Presynaptic MF glomeruli from lobes I (top) and VII (bottom) are visualized by VGluT1 and VGluT2. The reduction in MF size is consistent regardless of the lobe. Rosettes are generally labeled with either VGluT1 or VGluT2 and occasionally with both markers consistent with them being innervated by a single synaptic input from different neuronal cell types. C, Quantification of the size of VGluT1/VGluT2 clusters (Pclowt/wt $=22.73 \pm 0.896$ $\mu \mathrm{m}^{2}, n=4$ animals; $P$ clo ${ }^{g t / g t}=9.46 \pm 0.2899 \mu \mathrm{m}^{2}, n=4$ animals; unpaired $t$ test, $\left.t_{(6)}=14.1,{ }^{* * * *} p<0.0001\right) . \boldsymbol{D}, \mathbf{E}$, Histograms show the distribution of puncta sized $5-50 \mu \mathrm{m}^{2}(\boldsymbol{D})$ and $50-260 \mu \mathrm{m}^{2}(\boldsymbol{E})$. The shift of the data indicates that $P c 10^{g t / g t} \mathrm{MFs}$ have more smaller puncta $\left(5 \mu \mathrm{m}^{2}\right)$, whereas $P c l 0^{\mathrm{wt} / \mathrm{wt}}$ MFs have more larger puncta $\left(\right.$ up to $\left.260 \mu \mathrm{m}^{2}\right)$. Scale bars: $A, 20 \mu \mathrm{m}(\mathrm{zoom})$ $10 \mu \mathrm{m} \mathrm{B}, 50 \mu \mathrm{m} ;(\mathrm{zoom}), 20 \mu \mathrm{m}$. Error bars indicate SEM. Data points represent average puncta size per animal from images taken from lobes I, III, V, VII, and IX,4 sections per animal (C-E). Animals used in this experiment are as follows: wt7, wt8, wt10, wt11; k06, k07, k09, ko10. 

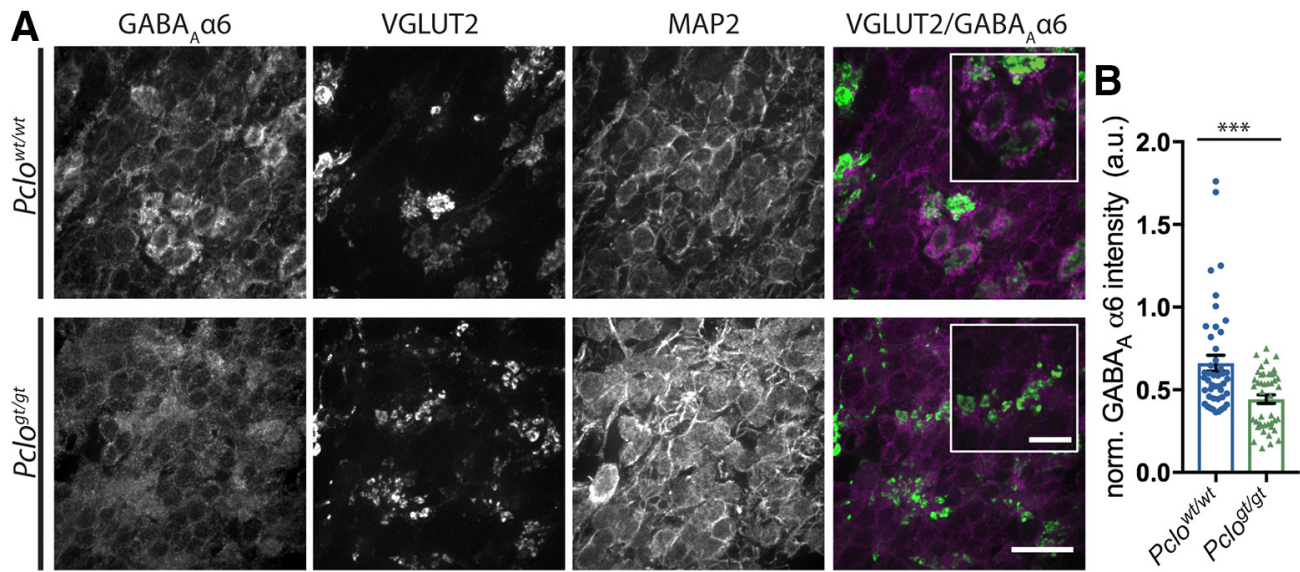

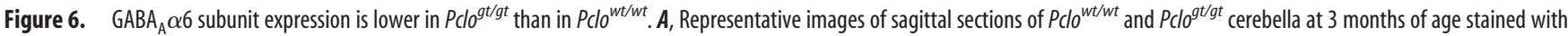
antibodies against the $\mathrm{GABA}_{\mathrm{A}}$ subunit $\alpha 6$, VGluT2, and MAP2. There are decreased levels of $\mathrm{GABA}_{A} \alpha 6$ in the GCL of $P$ clo $0^{g t / g t}$ animals compared with $P$ Clowt/wt controls, quantified in $\boldsymbol{B}$. $\boldsymbol{B}$, Quantification of $\mathrm{GABA}_{A}$ subunit $\alpha 6$, measured by the average intensity of antibody staining in images taken from the GCL of the cerebellum, normalized to MAP2 antibody intensities for the same image $\left(P c 10^{\text {wt } / w t}=0.661 \pm 0.0479\right.$ a.u.,$n=45$ images from 3 individual animals; $P$ clogt/gt $=0.443 \pm 0.26$ a.u., $n=44$ images from 3 individual animals; Mann-Whitney $U$ test, $U=589$, $* * * p=$ 0.0009). Scale bars, $20 \mu \mathrm{m}$. Error bars indicate SEM. Data points represent images taken from 4 sections per animal. Animals used in this experiment are as follows: wt10, wt11, wt13; k09-k011.

subclusters of SVs with large 100-200 $\mu \mathrm{m}^{3}$ terminals (Jakab and Hámori, 1988). In sections from Pclo ${ }^{g t / g t}$ animals, each bouton appeared smaller in size and less organized, although they still appear to contact GC dendrites (Fig. 5A). This impression is further supported by sections immunostained with antibodies against VGluT1 and VGluT2. In these images, distinct VGluT1and/or VGluT2-positive clusters are observed in both $\mathrm{Pclo}^{w t / w t}$ and $P c l o^{g t / g t}$ animals, consistent with individual boutons or glomeruli (Fig. 5B). Here again, MF terminals from $\mathrm{Pclo}^{g t / g t}$ animals appeared to have much smaller VGluT1- or VGluT2-positive clusters (Fig. 5B, zoom). Quantifying the average size of the VGluT1- and VGluT2-positive clusters revealed that clusters of $P c l o^{w t / w t}$ cerebella were more than double the size compared with the same lobes in $\mathrm{Pclo}^{g t / g t}$ animals (Fig. $5 \mathrm{~B}, \mathrm{C}$ ). However, this effect was not lobe-specific as it was observed in all lobes and not just in those receiving pontocerebellar afferents (Fig. 5B). These findings suggest that defects in the formation of large robust MF glomeruli in $\mathrm{Pclo}^{g t / g t}$ cerebella are a common feature shared by MF afferents arising from the pons and other brainstem nuclei, and may reflect aberrant signaling during development between GCs and these neurons.

Quantitatively, the spread of distribution of VGluT1 and VGluT2 cluster sizes is far more shifted toward smaller cluster sizes in $\mathrm{Pclo}^{g t / g t}$, with mutant cerebellar displaying $\sim 45 \%$ more small synaptic clusters of $5 \mu \mathrm{m}^{2}$ in size than $P c l o{ }^{w t / w t}$ counterparts (Fig. $5 D)$. At the larger end of the scale, cluster sizes $>50 \mu \mathrm{m}^{2}$ were much more frequent for Pclo ${ }^{w t / w t}$ than $P c l o^{g t / g t}$ cerebella (Fig. $5 E$ ).

In addition to excitatory input, MF glomeruli are also modulated by GABAergic inhibition via cerebellar Golgi cells, which offer regulatory feedback to the complex, as they themselves are excited by GCs (Maex and De Schutter, 1998). In principle, smaller MF terminals seen in $\mathrm{Pclo}^{g t / g t}$ animals could represent less excitatory input into GCs. This may also reduce excitatory drive onto PCs via PFs from GCs, as well as perhaps inhibitory drive via the Golgi cells. One of the dominant receptors mediating inhibitory input from Golgi cells to GCs are the $\alpha 6$ subunit-expressing $\mathrm{GABA}_{\mathrm{A}}$ receptors, which are highly concentrated within MF glomeruli (Nusser et al., 1996). Sagittal sections immunostained with antibodies against VGluT2 and $\alpha 6$ subunits revealed that the $\mathrm{GABA}_{\mathrm{A}} \alpha 6$ receptor subunit is only weakly expressed in $\mathrm{Pclo}^{g t / g t}$ MF rosettes, whereas in $P c l o^{w t / w t}$, it nicely localizes to the synaptic complex (Fig. 6A). Antibody staining of the GC layer demonstrates higher intensity of $\mathrm{GABA}_{\mathrm{A}} \alpha 6$ antibody staining in $\mathrm{Pclo}^{w t / w t}$ compared with $\mathrm{Pclo}^{g t / g t}$ (mean intensity: $\mathrm{Pclo}^{\mathrm{wt} / \mathrm{wt}}=$ 0.661 arbitrary units [a.u.]; $P c l o^{g t / g t}=0.443$ a.u.) (Fig. $\left.6 B\right)$. However, KO models of $\mathrm{GABA}_{\mathrm{A}} \alpha 6$ (Homanics et al., 1997) do not display $\mathrm{PCH} 3$ phenotypes or alterations in the anatomy of the cerebellum. Therefore, $\mathrm{GABA}_{\mathrm{A}} \alpha 6$ downregulation can be attributed to Piccolo loss, but not causal for the $\mathrm{PCH} 3$ phenotypes.

These data suggest that the loss of Piccolo not only affects the size of MF inputs into the cerebellum, but also gene expression and therefore inhibitory drive within each glomerulus, a condition that could relate to the maturation of these structures and/or their functionality, a situation that could adversely affect cerebellar function.

\section{Ultrastructural analysis of MF glomeruli in}

Pclo $^{\text {gt/gt }}$ cerebellum

The reduced area of VGluT1 and VGluT2 clusters within Pclo ${ }^{g t / g t}$ MF terminals (Fig. 5) could be due to a reduction in size of MF glomeruli themselves and/or in the number of VGluT1/2-positive SVs per bouton. To explore these options, we investigated MF glomeruli in $P c l o^{w t / w t}$ and $P c l o^{g t / g t}$ cerebella using EM. Analysis of ultrathin cerebellar brain sections from 3-month-old rats revealed that the average size of $P c o^{g t / g t}$ glomeruli was significantly smaller than $P c l o{ }^{w t / w t}$ glomeruli (mean glomeruli area: $P c l o{ }^{w t / w t}=$ $\left.34.59 \mu \mathrm{m}^{2} ; P_{c l o}^{g t / g t}=18.82 \mu \mathrm{m}^{2}\right)($ Fig. $7 \mathrm{~A}, \mathrm{C})$, whereas the number of SVs per area remained unchanged (Fig. $7 F$ ). These findings provide evidence for an overall reduction of SVs at each MF terminal. Furthermore, the complexity of the glomeruli indicated by a $\mathrm{P} 2 \mathrm{~A}$ value, measuring the ratio of perimeter per area, was significantly reduced by $35.8 \%$ (Fig. $7 B, E$ ). The size of the presynaptic area was also reduced in $\mathrm{Pclo}^{\text {gt/gt }}$ cerebellar sections (mean presynaptic area: $P c l o^{w t / w t}=17.73 \mu \mathrm{m}^{2} ; P_{c l o}{ }^{g t / g t}=11.69$ $\mu \mathrm{m}^{2}$ ) (Fig. $7 B, D$ ). However, the number of AZs present at each glomerulus was still proportional to their size, as $P c l o^{w t / w t}$ and Pclo ${ }^{g t / g t}$ had a similar number of AZs per glomerular area (Fig. $7 \mathrm{~B}, \mathrm{H})$. This leads to a reduction in the total number of AZs per presynaptic profile (mean AZs per terminal: $\mathrm{Pclo}^{w t / w t}=10.00$; $\left.P c l o^{g t / g t}=6.75\right)($ Fig. $7 \mathrm{~B}, \mathrm{~J})$. Piccolo LOF did not, however, affect the length of the AZ itself (Fig. 7G,I). These data indicate that the overall output of the MF glomeruli could be reduced in $\mathrm{Pclo}^{\mathrm{gt} / \mathrm{gt}}$ 
A

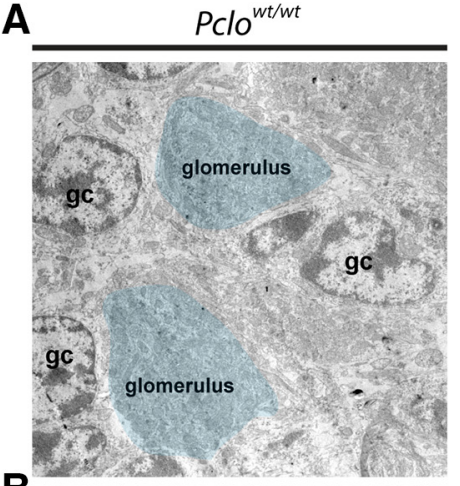

B

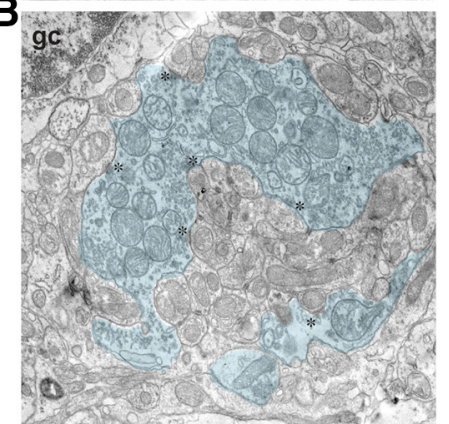

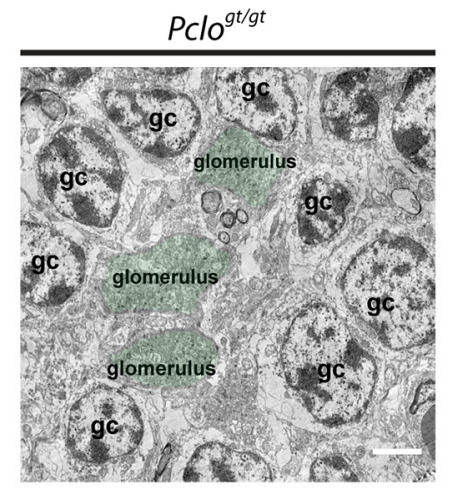

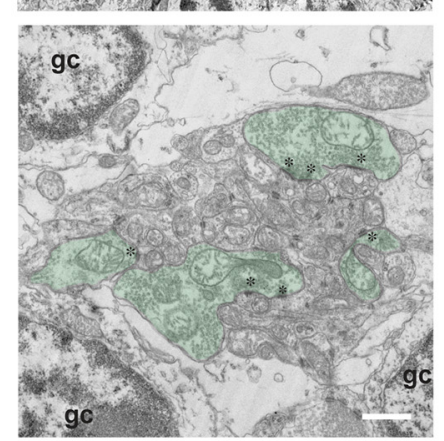

C

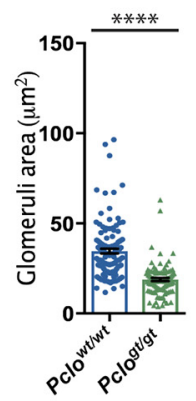

$E$

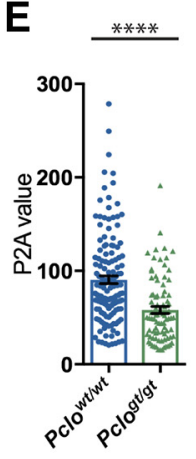

D

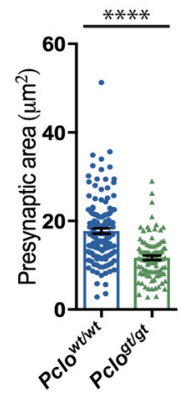

$F$

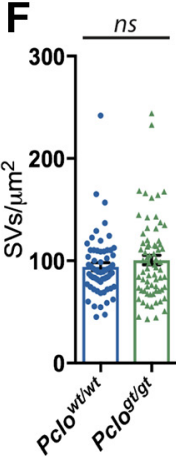

G
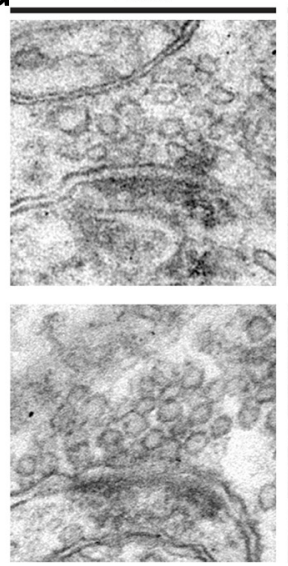

Pclo ${ }^{g t / g t}$
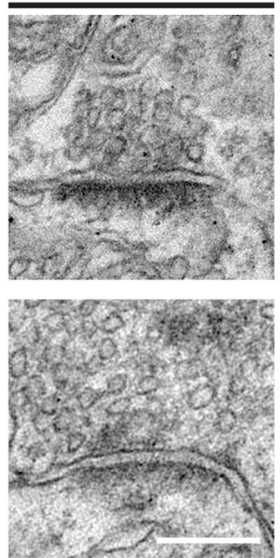
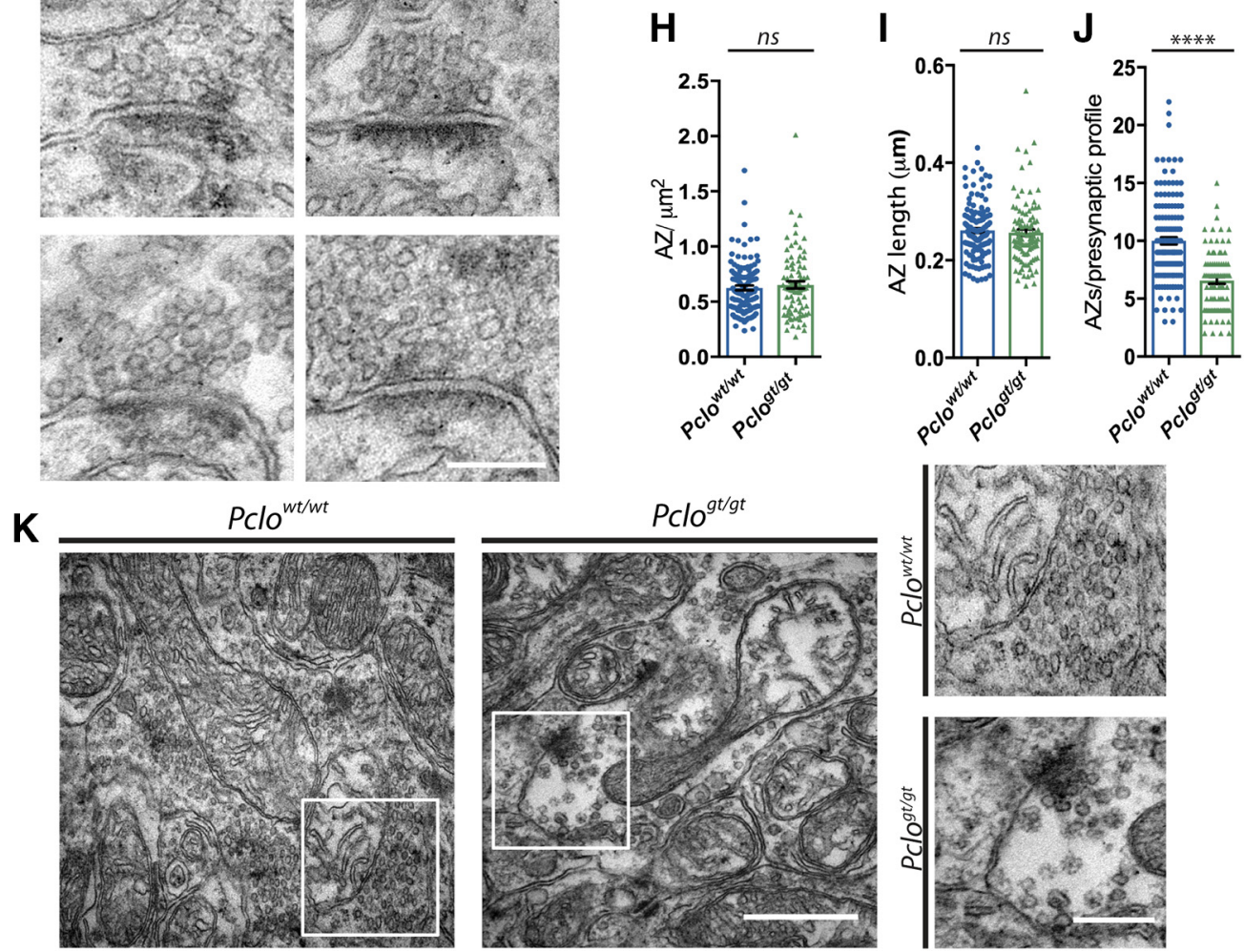
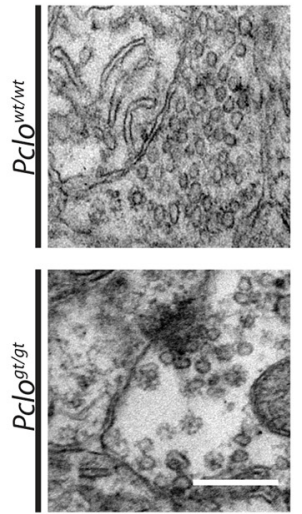

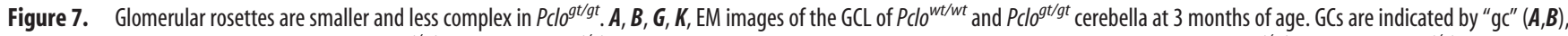
cerebellar glomeruli are highlighted in blue $\left(P c 10^{w t / w t}\right)$ and green $\left(P c / 0^{g t / g t}\right)(A)$, and the presynaptic terminals of cerebellar MF are highlighted in blue $\left(P c 10^{w t / w t}\right)$ and green $\left(P c 10^{g t / g t}\right)(B)$. Asterisks

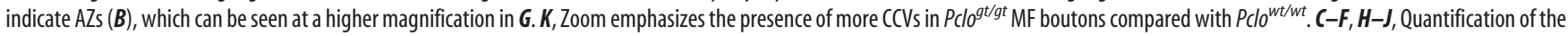
size of glomeruli $(\boldsymbol{C})$, the size of the MF presynapse $(\boldsymbol{D})$, the complexity (squared perimeter/presynaptic area) of the MFbouton $(\boldsymbol{E})$, the density of $S V s(\boldsymbol{F})$, the number of AZs per area $(\boldsymbol{H})$, the AZ length

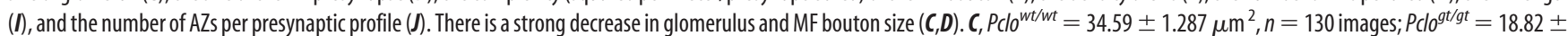
$0.853 \mu \mathrm{m}^{2}, n=103$ images $; n=3$ independent experiments; Mann-Whitney Utest, $U=1733 .{ }^{* * *} p<0.0001 . D, P c 10^{\text {wt } / \mathrm{wt}}=17.73 \pm 0.603 \mu \mathrm{m}^{2}, n=141 \mathrm{images} ; P c l 0^{g t / g t}=11.69 \pm 0.497$ $\mu \mathrm{m}^{2}, n=95$ images $n=3$ independent experiments; Mann-Whitney $U$ test $U=3057$. ${ }^{* * * *} p<0.0001 . E, P c l 0^{\text {wt } / \text { wt }}=90.5 \pm 4.089, n=141$ images; $P$ clo $0^{g t / g t}=58.1 \pm 3.818, n=84$ images; $n=3$ independent experiments; Mann-Whitney $U$ test $, U=3373 .{ }^{* * * *} p<0.0001 . F, P c l 0^{\text {wt } / w t}=94.24 \pm 3.853 \mathrm{SVs} / \mu \mathrm{m}^{2}, n=62 \mathrm{images} ; P c l 0^{g t / g t}=100.7 \pm 4.705 \mathrm{SVs} / \mu \mathrm{m}^{2}, n=$ 71 images; $n=3$ independent experiments; Mann-Whitney $U$ test, $U=2042 . p=0.476 . \boldsymbol{H}, P$ cllot ${ }^{\text {wt }}=0.625 \pm 0.021, n=121$ images; $P$ clogt/gt $=0.652 \pm 0.032, n=84$ images; $n=3$ independent experiments; Mann-Whitney $U$ test, $U=4942 . p=0.7390 . I, P c l 0^{\text {wt } / w t}=0.261 \pm 0.000487 \mu \mathrm{m}, n=129$ images; Pclogt/gt $=0.257 \pm 0.00653 \mu \mathrm{m}$, (Figure legend continues.) 

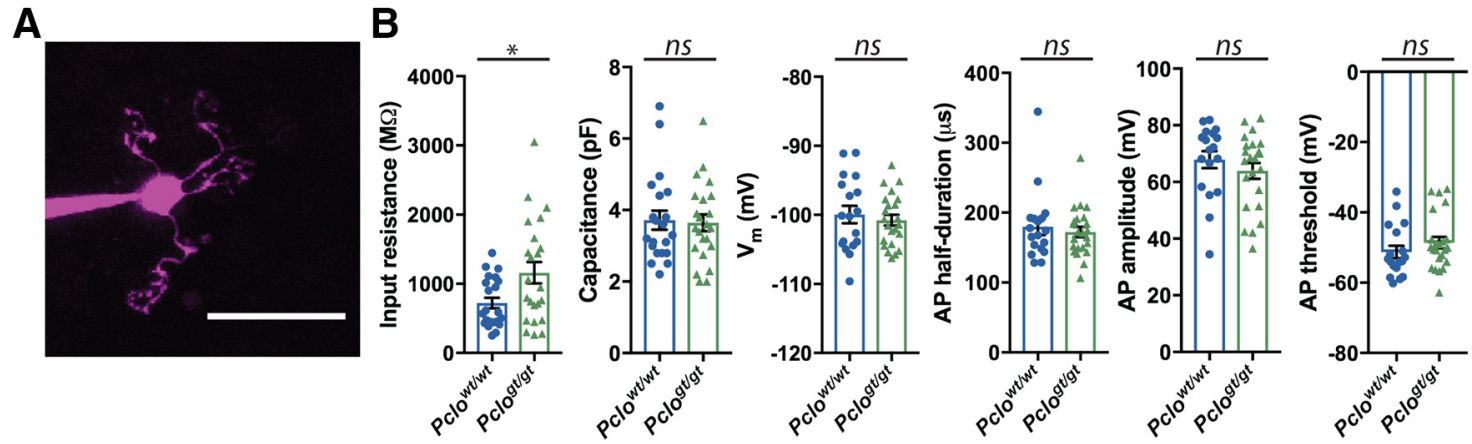

C
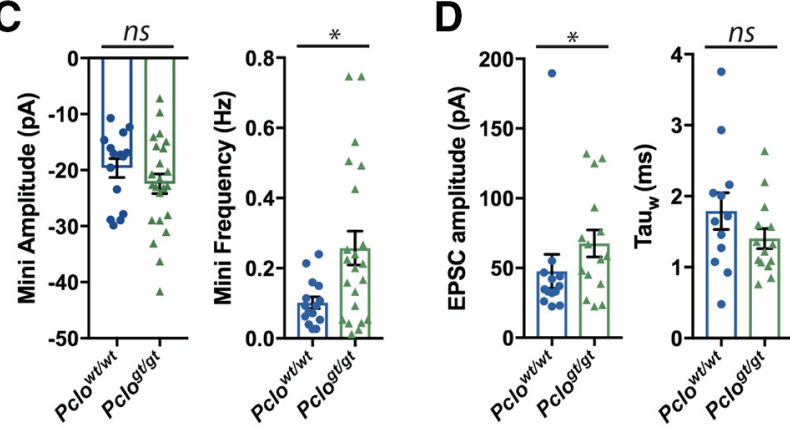

Pclowt/wt

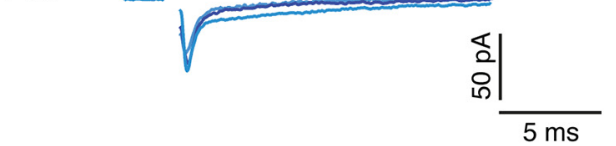

Pclogt/gt

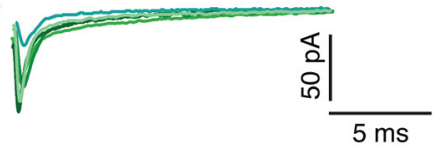

Figure 8. Physiological assessment of MF boutons. $A$, Example two-photon image of a cerebellar GC from a $P$ clogt/gt rat filled with ATTO dye. $B$, Average data of biophysical properties of GCs for

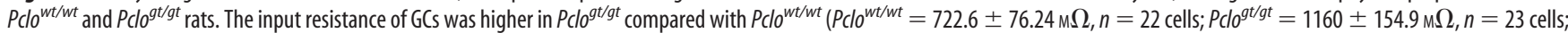
$n=3$ rats per genotype; Mann-Whitney $U$ test, $\left.U=165,{ }^{*} p=0.0462\right)$. Whereas no differences were found in capacitance $\left(P c l 0^{\text {wt } / \mathrm{wt}}=3.717 \pm 0.269 \mathrm{pF}, n=21 \mathrm{cells} ; P\right.$ cllot/gt $=3.643 \pm 0.231$ $\mathrm{pF}, n=23$ cells; $n=3$ rats per genotype; Mann-Whitney $U$ test $U=236.5, p=0.912)$, resting membrane potential $\left(V_{\mathrm{m}}\right)\left(P c l 0^{\mathrm{wt} / \mathrm{wt}}=-99.96 \pm 1.261 \mathrm{mV}, n=18 \mathrm{cells} ; P \mathrm{cl} 0^{g t / g t}=-100.8 \pm\right.$ $0.786 \mathrm{mV}, n=23$ cells; $n=3$ rats per genotype; Mann-Whitney $U$ test, $U=187, p=0.612)$, the half-duration of the AP $\left(P c l 0^{\text {wt } / w t}=179.7 \pm 11.84 \mu \mathrm{s}, n=18 \mathrm{cells} ; P\right.$ clo $0^{\mathrm{gt} / g t}=172.2 \pm 7.366$ $\mu s, n=23$ cells; $n=3$ rats per genotype; Mann-Whitney $U$ test, $U=200, p=0.866)$, the amplitude of the AP $\left(P c l 0^{w t / w t}=67.85 \pm 3.016 \mathrm{mV}, n=18\right.$ cells; $P c 10^{g t / g t}=63.92 \pm 2.761 \mathrm{mV}, n=$ 23 cells; $n=3$ rats per genotype; Mann-Whitney $U$ test, $U=166, p=0.291)$, and the voltage threshold to elicit an AP (Pclo ${ }^{\mathrm{wt} / \mathrm{wt}}=-51.27 \pm 1.748 \mathrm{mV}, n=18 \mathrm{cellls} ; P$ clo ${ }^{g t / g t}=-48.6 \pm 1.659$ $\mathrm{mV}, n=23$ cells; $n=3$ rats per genotype; Mann-Whitney $U$ test, $U=153 p=0.162) . C$, mEPSCs from $P$ clo ${ }^{g t / g t} \mathrm{GCs}$ were not different in their amplitude $\left(P c / 0^{\text {wt } / w t}=-19.62 \pm 1.682 \mathrm{pA}, n=\right.$ 15 cells from 2 rats; $P$ c $10^{g t / g t}=-22.44 \pm 1.765 \mathrm{pA}, n=23$ cells from 3 rats; Mann-Whitney $U$ test, $\left.U=136, p=0.286\right)$ but in their frequency $\left(P c l 0^{\mathrm{wt} / \mathrm{wt}}=0.102 \pm 0.0167 \mathrm{~Hz}, n=15\right.$ cells; $P c 10^{g t / g t}=0.257 \pm 0.0481 \mathrm{~Hz}, n=22$ cells; $n=3$ rats per genotype; Mann-Whitney $U$ test, $\left.U=98.5,{ }^{*} p=0.0392\right) . D$, EPSCs from GCs measured after stimulation of single MFs were increased

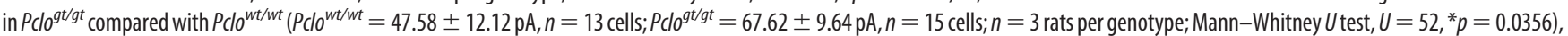
whereas the decay of the EPSCs was not altered $\left(P c l 0^{w t / w t}=1.79 \pm 0.258 \mathrm{~ms}, n=12\right.$ cells; $P$ clo $0^{\mathrm{gt} / g t}=1.404 \pm 0.141 \mathrm{~ms}, n=14$ cells; $n=3$ rats per genotype; Mann-Whitney $U$ test, $U=60$, ${ }^{*} p=0.231$. Right, Example traces of evoked EPSCS, as quantified in D, in response $1 \mathrm{~Hz}$ stimulation in the presence of $20 \mu \mathrm{m}$ SR95531 and $40 \mu \mathrm{m} \mathrm{D}-A P V$. Scale bar: $A, 20 \mu \mathrm{m}$. Error bars indicate SEM. Data points represent individual cells from 3 rats per genotype. Animals used in this experiment are as follows: wt18-wt20; ko17-ko19. ns, not significant.

cerebella. Intriguingly, we also noticed an accumulation of clathrin-coated vesicles (CCVs) in the terminals of $P c l o^{g t / g t} \mathrm{MFs}$ (Fig. $7 \mathrm{~K}$ ). This phenotype resembles recent findings from hippocampal synapses from $P c l^{g t / g t}$ animals, which revealed defects in the formation of endosomal membranes and an overall reduction in SV number (Ackermann et al., 2019) and suggest possible changes in the recycling of SVs within MF boutons after fusion with the plasma membrane.

\section{Piccolo loss alters GC properties and MF to GC synaptic transmission}

The anatomical and morphological changes observed in MF boutons lacking Piccolo are predicted to not only represent altered afferent input into the cerebellum from the pons and other brainstem nuclei, but also altered cerebellar function. As an initial test of this hypothesis, we performed whole-cell current-clamp recordings of cerebellar GCs from acute P90 rat cerebellar slices. A

\section{$\leftarrow$}

(Figure legend continued.) $n=101$ images; $n=3$ independent experiments; Mann-Whitney $U$ test, $U=5963 . p=0.271 . J, P c l 0^{w t / w t}=10 \pm 0.304, n=141$ images; $P$ clotot $/ 9 t=6.571 \pm$ $0.295, n=84$ images; $n=3$ independent experiments; Mann-Whitney $U$ test, $U=2650$. ${ }^{* * * *} p<0.0001$. Scale bars: $\boldsymbol{A}, 2.5 \mu \mathrm{m} ; \boldsymbol{B}, 1 \mu \mathrm{m} ; \boldsymbol{G}, 125 \mathrm{~nm} ; \boldsymbol{K}, 500 \mathrm{~nm} ; \boldsymbol{K}(\mathrm{zoom}), 250 \mathrm{~nm}$. Error bars indicate SEM. Animals used in this experiment are as follows: wt15-wt17; k014-ko16. ns, not significant. two-photon image of a typical cerebellar GC from $P c l o^{g t / g t}$ filled with ATTO dye reveals a normal radial arrangement of its dendrites as they project their claws into MF glomeruli (Fig. 8A). An analysis of the intrinsic biophysical properties of these cells revealed that some GC properties differed between $P c l o^{g t / g t}$ and $P c l 0^{w t / w t}$ animals. Specifically, no changes were detected in either the capacitance or the membrane potential of these cells, but the input resistance was significantly increased by $60 \%$ (Fig. $8 B$ ). Since these experiments were performed in the presence of $\mathrm{GABA}_{\mathrm{A}}$ receptor blockers, the decreased shunting inhibition mediated by tonic activation of $\alpha 6$-subunit-containing $\mathrm{GABA}_{\mathrm{A}}$ receptors (Brickley et al., 1996; Nusser et al., 1998) is expected to further increase the difference in input resistance. Yet, there was also no change in the amplitude, threshold of activation, or duration of APs fired by these cells (Fig. $8 B$ ), indicating unaltered active membrane properties. Examining the frequency and amplitude of spontaneous mEPSCs of these GCs revealed a dramatic increase of $151 \%$ in the frequency of these events in Pclo ${ }^{g t / g t}$ slices with no change in mEPSC amplitudes (Fig. $8 \mathrm{C}$ ). These data suggest that, on average, each excitatory synapse formed on to these GC dendrites has normal levels of postsynaptic AMPA-type glutamate receptors. The change in frequency could either be due to an increase in the number of MF boutons contacting GC dendrites and/or an increase in the release probability of MF boutons. Consistently, the average amplitude of the evoked EPSCs 
A
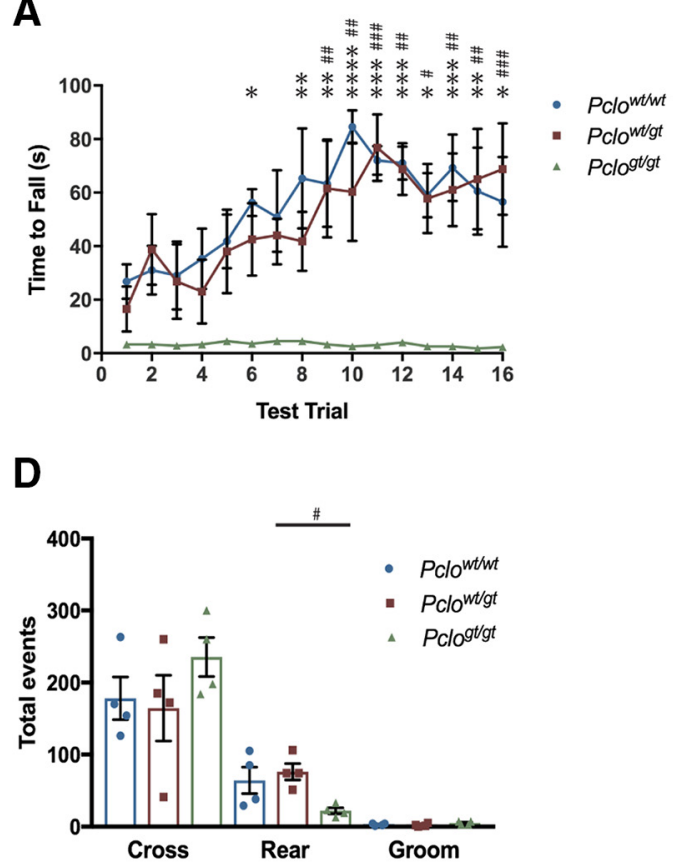

B

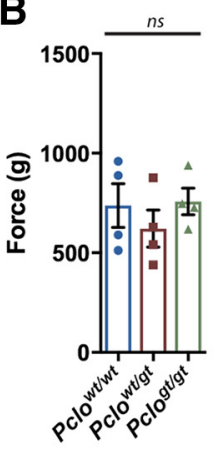

E

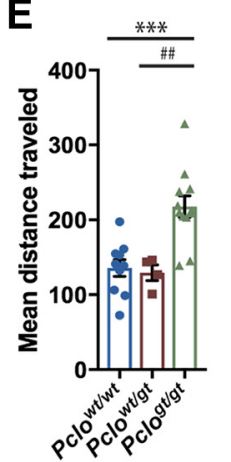

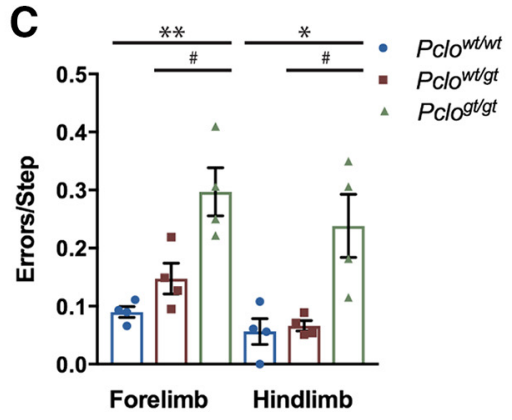

$\mathbf{F}$

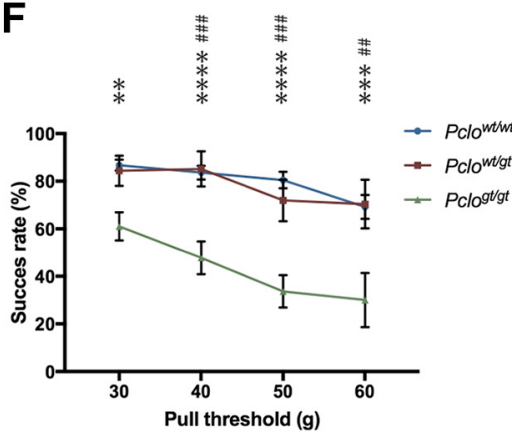

Figure 9. Behavioral outcome of Piccolo LOF resembles $P C H 3$ symptoms. $A$, Rotarod performance for $P$ clo ${ }^{w t / w t}, P C l 0^{w t / g t}$, and $P$ clo ${ }^{g t / g t}$ rats for 16 trials over $4 \mathrm{~d}$. $P$ Clo ${ }^{g t / g t}$ rats fell significantly faster than $P$ clo ${ }^{w / w}$ on Trials 6 and 8 and faster than both $P$ clo ${ }^{w t / w t}$ and $P$ Clo ${ }^{w t / g t}$ on Trials $9-16$. B, Grip strength task for $P$ clo ${ }^{w t / w t}$, $P$ clo ${ }^{w t / g t}$, and $P$ clo $0^{g t / g t}$ rats for 9 trials over $2 \mathrm{~d}$. No differences were found for forelimb grip strength between the groups. C, Ladder walk task for $P$ clowt/wt, $859 P \mathrm{Cl} 0^{w t / g t}$, and $P$ clo ${ }^{g t / g t}$ rats for 3 trials over $1 \mathrm{~d}$. $P$ clo ${ }^{g t / g t}$ rats had a higher rate of stepping errors (ladder rung foot slips/misses) than $P$ clowt/wt and $P$ clowt/gt rats. D, Open field task for $P$ clowt/wt,$P c l 0^{w t / g t}$, and $P$ clo ${ }^{g t / g t}$ rats for 1 trial each over $1 \mathrm{~d}$. $P$ clo ${ }^{g t / g t}$ rats performed fewer rearing behaviors than $P$ clo ${ }^{w t / w t}$ and significantly less than $P$ clowt/gt rats in the perimeter sectors of the arena. Other behaviors, such as crossing the open field and grooming, were not different between the three groups. Animals used in the above experiments are as follows: wt21-wt24; he1-he4; k020-k023. E, Locomotor activity of $P$ clowt/wt,$P c l 0^{w t / g t}$, and $P$ clo ${ }^{g t / g t}$ rats during the $12 \mathrm{~h}$ dark phase. $P$ clo ${ }^{g t / g t}$ rats covered a $>50 \%$ longer distance than $P$ clowt/wt and $P$ clo ${ }^{w t / g t}$ rats. Data points are individual means over 15 nights. F, Performance of $P$ clowt/wt,$P$ clo ${ }^{w t / g t}$, and $P$ clo ${ }^{g t / g t}$ rats during the isometric pull-task (handle position $11 \mathrm{~mm}$ inside the cage). Only 4 of $11 P c l 0^{g t / g t}$ rats succeeded at the $60 \mathrm{~g}$ force threshold, and $P$ clo ${ }^{g t / g t}$ rats pulled with a significantly lower success rate at all force thresholds compared with $P$ clowt/wt and $P c l 0^{w t / g t}$. Error bars indicate SEM. Data points represent individual rats. For statistical analysis, see Table 1. Animals used in the above experiments are as follows: wt25-wt34; he5-he8; k024-k035. ${ }^{*},{ }^{* *},{ }^{* * *},{ }^{* * *}$ describe significance between $P\left(10^{w t / w t}\right.$ and $P c l 0^{g t / g t}$ and \#, \#\#, \#\#\# describe significance between $P c l 0^{w t / w t}$ and $P c l 0^{g t / g t}$.

was increased in $P c l o^{g t / g t}$ animals (mean EPSC amplitude: $P c l o^{w t / w t}=$ $\left.47.58 \mathrm{pA} ; P_{c l o} o^{g t / g t}=67.62 \mathrm{pA}\right)$, with no change in the weighted time constant $\left(\tau_{\mathrm{w}}\right)$ (Fig. $8 D$ ). Given that the amplitudes of the mEPSCs are not changed, these results suggest that there is a higher number of synaptic connections between MF boutons and the dendrites of GCs. Together, these data indicate that, in addition to changes in cell number, the loss of Piccolo in the cerebellum and brainstem is associated with changes in the morphology and function of GCs and their MF input.

\section{Behavioral and motor defects in Piccolo KO rats}

The motor difficulties reported in humans with PCH3 (Zelnik et al., 1996; Durmaz et al., 2009) and the anatomical changes observed in the cerebellum and brainstem of rats lacking Piccolo predict altered motor function in these animals. To test this hypothesis, $\mathrm{Pclo}^{w t / w t}, \mathrm{Pclo}^{w t / g t}$, and $\mathrm{Pclo}^{g t / g t}$ rats were monitored for their motor abilities. In rotarod tasks, $P c l o^{g t / g t}$ rat performance was significantly reduced compared with $P c l o^{w t / w t}$ and $P c l o^{w t / g t}$ rats (Fig. 9A). Specifically, whereas $P c l o^{w t / w t}$ and $P c l o{ }^{w t / g t}$ rats exhibited increasing performance levels regarding the ability to stay on the rotarod over time, $P c o^{g t / g t}$ rats showed no indication of being able to adapt to the task. Pclo ${ }^{g t / g t}$ rats were less adept at staying on the task apparatus once rod rotation was initiated (mean time at trial 16: $\mathrm{Pclo}^{w t / w t}=56.5 \mathrm{~s} ; \mathrm{Pclo}^{w t / g t}=68.75 \mathrm{~s}$; $\left.P c l o^{g t / g t}=2.25 \mathrm{~s}\right)($ Fig. 9A; Table 1). Intriguingly, no differences in forelimb grip strength were scored between Piccolo genotypes (Fig. 9B; Table 1), indicating that $P c l o^{g t / g t}$ rats showed lack of motivation and/or impaired coordination rather than muscle strength.

In addition to deficits in motor ability, $P c l o^{g t / g t}$ rats displayed an increased frequency in front and rear foot stepping errors during ladder rung walking compared with $\mathrm{Pclo}^{w t / w t}$ and $\mathrm{Pclo}{ }^{w t / g t}$ rats (mean forelimb error/step: $P c l o^{w t / w t}=0.09 ; P_{c l o}{ }^{w t / g t}=0.148$; $P c l o^{g t / g t}=0.29$; mean hindlimb error/step: $P c l o^{w t / w t}=0.0563$; $P c l o{ }^{w t / g t}=0.0663 ;$ Pclo $\left.^{g t / g t}=0.238\right)($ Fig. $9 C$; Table 1). In open field tests, no significant differences were recorded between $P c l o{ }^{w t / w t}$, $\mathrm{Pclo}^{w t / g t}$, and $\mathrm{Pclo}{ }^{g t / g t}$ rats for peripheral line crossing or selfgrooming events (Fig. 9D; Table 1). However, $P c l o^{g t / g t}$ rats displayed twofold to threefold decreases in peripheral rearing events compared with $P c l o^{w t / w t}$ and $P c l o^{w t / g t}$ animals (Fig. 9D). Deficits in $\mathrm{Pclo}^{\mathrm{gt} / g t}$ rat performance during rotarod, ladder rung, and open field tests compared with $P c l o^{w t / w t}$ and $P c l o^{w t / g t}$ (Fig. $9 A-D$ ) reflected recessive traits, consistent with dysfunction in proprioceptive sensation and motor control (Curzon et al., 2009).

Alongside traditional tests for motor coordination, we tested the behavior of $P c l o^{w t / w t}, P c l o o^{w t / g t}$, and $P c l o{ }^{g t / g t}$ rats in a home cage setup, the OptiMan system, where animals can be monitored without interference from experimenters. Rats were tagged with a RFID chip that allowed for tracking of locomotor activity while they were also required to complete an isometric pull task that requires precise and finely controlled movements. In the home cage, the $\mathrm{Pclo}^{g t / g t}$ rats were more active and covered almost twice the distance than $\mathrm{Pclo}^{w t / w t}$ rats (Fig. 9E) during each measurement. In the isometric pull task, performance of $P c{ }^{g t / g t}$ rats was 
Table 1. Supplementary statistical information supporting Figure $9^{a}$

\begin{tabular}{|c|c|c|c|c|c|c|c|c|c|c|}
\hline Behavioral test & $\begin{array}{l}\text { Trial/ } \\
\text { event }\end{array}$ & $P c 10^{w t / w t}$ & $n$ & Pclott/gt & $n$ & $P\left(1 g^{g t / g t}\right.$ & $n$ & $\begin{array}{l}w t / w t \text { versus wt/gt (df), } \\
t \text { value, } p \text { value }\end{array}$ & $\begin{array}{l}w t / w t \text { versus } g t / g t(d f), \\
t \text { value, } p \text { value }\end{array}$ & One-way ANOVA \\
\hline Rotarod performance: & 1 & $26.75 \pm 6.486$ & 4 & $16.5 \pm 8.431$ & 4 & $3.25 \pm 0.75$ & 4 & $(144), 0.681,>0.9999$ & $(144), 1.561,>0.9999$ & \\
\hline \multirow[t]{10}{*}{ Time to fall (s) } & 2 & $3 \pm 9.138$ & 4 & $38.75 \pm 13.231$ & 4 & $3.25 \pm 0.479$ & 4 & $(144), 0.515,>0.9999$ & $(144), 1.843,>0.9999$ & \\
\hline & 4 & $35.25 \pm 11.25$ & 4 & $23 \pm 11.979$ & 4 & $3.25 \pm 0.479$ & 4 & $(144), 0.814,>0.9999$ & $(144), 2.126,>0.9999$ & \\
\hline & 5 & $41.75 \pm 10.053$ & 4 & $38 \pm 15.647$ & 4 & $4.5 \pm 1.041$ & 4 & $(144), 0.249,>0.9999$ & $(144), 2.474,0.6963$ & \\
\hline & 6 & $56.25 \pm 5.023$ & 4 & $42.5 \pm 13.531$ & 4 & $3.5 \pm 0.5$ & 4 & $(144), 0.1913,>0.9999$ & $(144), 3.504,0.0293$ & \\
\hline & 9 & $63.25 \pm 16.163$ & 4 & $61.5 \pm 18.319$ & 4 & $3.25 \pm 0.75$ & 4 & $(144), 0.116,0.0079$ & $(144), 3.986,0.0051$ & \\
\hline & 10 & $84.5 \pm 6.225$ & 4 & $60.25 \pm 18.396$ & 4 & $2.5 \pm 0.5$ & 4 & (144), $1.611,0.0089$ & $(144), 5.447,0.0001$ & \\
\hline & 11 & $72 \pm 5.339$ & 4 & $76.75 \pm 12.419$ & 4 & $3 \pm 0.408$ & 4 & $(144), 0.316,0.001$ & $(144), 4.583,0.0005$ & \\
\hline & 12 & $71 \pm 6.178$ & 4 & $68.75 \pm 9.681$ & 4 & $4 \pm 0.707$ & 4 & $(144), 0.15,0.0015$ & $(144), 4.451,0.0008$ & \\
\hline & 13 & $59 \pm 8.297$ & 4 & $57.75 \pm 12.99$ & 4 & $2.5 \pm 0.5$ & 4 & $(144), 0.083,0.0164$ & $(144), 3.753,0.0121$ & \\
\hline & 14 & $69.25 \pm 12.479$ & 4 & $61 \pm 13.638$ & 4 & $2.5 \pm 0.645$ & 4 & $(144), 0.548,0.0074$ & $(144), 4.434,0.0009$ & \\
\hline Ladder walk: HL errors/step & & $0.0563 \pm 0.0221$ & 4 & $0.0663 \pm 0.00877$ & 4 & $0.238 \pm 0.0543$ & 4 & $\# p=0.0186$ & ${ }^{*} p=0.0135$ & \\
\hline \multirow[t]{3}{*}{ Open field task: total events } & Cross & $178.3 \pm 29.68$ & 4 & $164.5 \pm 45.51$ & 4 & $235.5 \pm 27.11$ & 4 & $p>0.9999$ & $p=0.8337$ & $F_{(2,9)}=1.154, p=0.828$ \\
\hline & Rear & $64.25 \pm 18.31$ & 4 & $76.25 \pm 11.3$ & 4 & $22 \pm 4.203$ & 4 & $p>0.9999$ & $p=0.1277$ & $F_{(2,9)}=5.069, p=0.0335$ \\
\hline & Groom & $2.75 \pm 0.75$ & 4 & $2 \pm 1.08$ & 4 & $5 \pm 1.155$ & 4 & $p>0.9999$ & $p=0.4494$ & $F_{(2,9)}=2.388, p=0.1473$ \\
\hline Mean distance traveled & & $135.7 \pm 11.25$ & 10 & $129.3 \pm 10.44$ & 4 & $217.8 \pm 14.33$ & 12 & $p>0.9999$ & ${ }^{* * *} p=0.0004$ & \\
\hline \multirow[t]{4}{*}{ Pull-task: force (success rate) } & $30 \mathrm{~g}$ & $86.722 \pm 2.3$ & 10 & $84.374 \pm 6.324$ & 4 & $61.044 \pm 5.928$ & 11 & $p>0.9999$ & ${ }^{* *} p=0.0018$ & \\
\hline & $40 \mathrm{~g}$ & $83.603 \pm 2.86$ & 10 & $85.136 \pm 7.373$ & 4 & $47.804 \pm 6.897$ & 10 & $p>0.9999$ & ${ }^{* * * *} p=0.0001$ & \\
\hline & $50 \mathrm{~g}$ & $80.49 \pm 3.442$ & 10 & $71.93 \pm 8.695$ & 4 & $33.647 \pm 6.802$ & 10 & $p>0.9999$ & ${ }^{* * * *} p=0.0001$ & \\
\hline & $60 \mathrm{~g}$ & $69.19 \pm 4.99$ & 10 & $70.409 \pm 10.181$ & 3 & $30.003 \pm 11.359$ & 4 & $p>0.9999$ & ${ }^{* * *} p=0.0004$ & \\
\hline
\end{tabular}

${ }^{a}$ Data are mean $\pm \mathrm{SEM}, F, n, \mathrm{df}, t$, and $p$ values for behavioral data as depicted in Figure 9.

significantly lower than the performance of $\mathrm{Pclo}^{w t / w t}$ rats, quantified using four different force thresholds (mean success rate at 60 g: $\left.P c l o o^{w t / w t}=69.186 ; P_{c l o}{ }^{w t / g t}=70.409 ; P c^{g t / g t}=30.003\right)$ (Fig. $9 F$ ). Together, $P c l o^{g t / g t}$ rats show clear motor deficits, very similar to symptoms seen in PCH3 patients (Rajab et al., 2003).

\section{Discussion}

Our study demonstrates that Piccolo LOF causes alterations in brain anatomy; the cerebrum, pons, brainstem, and cerebellum are severely reduced in size (Fig. 1). These changes are associated with reductions in cerebellar and pontine cell numbers and perturbations in cerebellar CF and MF afferents (Figs. 4, 5). These changes are predicted to adversely affect cerebellar function, supported by changes in synaptic transmission and motor control and the presence of seizures in Pclo $^{g t / g t}$ rats (Medrano et al., 2019). Interestingly, the changes in brain morphology resemble changes in children with $\mathrm{PCH} 3$ recently linked to a single nucleotide polymorphism in the Pclo gene (Ahmed et al., 2015). In addition to brain atrophy, patients with $\mathrm{PCH} 3$ exhibit seizures and cognitive and motor deficits.

Similar to patients with $\mathrm{PCH} 3, \mathrm{Pclo}^{g t / g t}$ rats have smaller cerebella, brainstems, and pontine nuclei. In the cerebellum, a striking change was the thickness of the ML and GCL (Fig. 3). The latter was associated with fewer total GCs, which could result in fewer PFs and a thinner ML. At present, it is unclear why there are fewer GCs in Pclogt/gt cerebella. During normal development, several factors, including Sonic hedgehog (Wallace, 1999; Miyashita et al., 2017) and Notch (Solecki et al., 2001), are known to control the proliferation of GCs. How Piccolo loss influences these and related signaling pathways is unclear, although alterations of neuronal gene expression, a consequence of Piccolo LOF (Hübler et al., 2012; Ivanova et al., 2015; Medrano et al., 2019), could contribute to the loss of GCs as a downstream effect.

In the ML, CF morphology and arrangement appear to be normal, although a higher innervation of PC dendrites by CFs can be observed (Fig. 4). One possible explanation for this hyperinnervation is that homeostatic changes to the network, such as heterosynaptic competition with PFs for PC dendrite territory (Hashimoto et al., 2009; Ichikawa et al., 2016), could contribute to these differences and therefore the functionality of the cerebellum. This could affect normal pruning mechanisms of CF during development.

Our study also revealed that pontine nuclei were dramatically decreased in size in $\mathrm{Pclo}^{g t / g t}$ animals. Afferent MFs from the pons and other brainstem nuclei are the primary excitatory input onto GCs, forming elaborate rosette synapses (Voogd and Glickstein, 1998). The smaller size of the pons suggests a net reduction in MF input into the cerebellum that appears to correspond to the reduced number of GCs (Fig. 3). However, it is unclear how the loss of Piccolo could influence the number of neurons in the pons.

Surprisingly, our histological studies revealed that MF boutons across multiple lobes of the cerebellum were reduced in size (Fig. 5). This was supported by our EM studies revealing that MF glomeruli have less SVs as they are severely reduced in size but with a similar SV density (Fig. 7). These observations suggest that the development/maturation of MFs from the pons and other brainstem nuclei is muted in Pclo ${ }^{g t / g t}$ animals. Additionally, electrophysiological changes (Fig. 8) indicate that the network has triggered compensatory changes to overcome a smaller size or impaired strength of MFs reaching the GC layer, for instance, by increasing both input resistance and release probability (Turrigiano, 2012). 
Electron micrographs demonstrate what could be disturbed synaptic integrity in $\mathrm{Pclo}^{g t / g t} \mathrm{MF}$ boutons with more CCVs being present (Fig. 7), suggesting that the loss of Piccolo may also affect SV recycling. These observations concur with recent findings by Ackermann et al. (2019) of hippocampal synapses from $\mathrm{Pclo}^{g t / g t}$ animals, which revealed that Piccolo LOF had a dramatic effect on the recycling of SV proteins through a block in the formation of early endosomes from endocytic vesicles, due to defects in the activation of Rab5 via a Piccolo-dependent synaptic loss of Pra1. Although beyond the current study, it is likely that this endocytic defect in the recycling of SV proteins could also contribute to altered size and function of MF synapses, especially given their high-frequency transmission and therefore need for high SV turnover (Byczkowicz et al., 2018).

By several measures mentioned above, it would appear that the MF glomeruli are less mature. Key regulators of MF maturation are members of the Wnt family, a group of target-derived factors that accelerate neuronal maturation or induce synapse formation (Scheiffele, 2003; Waites et al., 2005). Interestingly, mouse cerebellar MFs lacking Wnt7a show a similar reduction in MF size and complexity, as observed in $P c l o^{g t / g t}$ (Hall et al., 2000). However, it is important to note that, in the case of Wnt7a loss, MF synapse size catches up to WT size by age $\mathrm{P} 15$, which points to a lag in maturation and does not seem to be the case in $P c l o^{g t / g t}$. KO of Disheveled1 (Dvl1), a downstream target of Wnt (Salinas and Zou, 2008), also shows a reduction in MF cluster size, indicating that presynaptic Dvl1 is a necessary step in the Wnt signaling cascade, underscoring the importance of these proteins for MF-GC synapse formation (Ahmad-Annuar et al., 2006). Like Dvl1, Piccolo is located at the AZ of the presynaptic terminal and regulates F-actin assembly and synaptic transmission through its interaction with Daam1 and Profilin (Wagh et al., 2015). Daam1 is a formin and a known regulator/interaction partner of Dvl1 (Gao and Chen, 2010). It is thus possible that, in the absence of Piccolo, proper Wnt signaling is disrupted, altering the maturation of MF-GC synapses. Initial analysis of $\mathrm{Pclo}^{g t / g t}$ transcripts reveals that Wnt expression is changed (Medrano et al., 2019). Moreover, Wnt3a was found to increase Piccolo RNA and protein levels in mouse hippocampal neurons (Martinez et al., 2019), supporting a relationship between Piccolo and Wnt ligands. Thus, there appear to be at least two possible mechanisms that could contribute to smaller MF boutons in Piccolo KO rats: defects in SV recycling and Wnt signaling. Clearly, further studies are needed to explore these options.

GCs also receive inhibitory modulation from Golgi cells (Eccles et al., 1966); it is well appreciated that $\mathrm{GABA}_{\mathrm{A}}$ receptors at the MF synapse contain $\alpha 6$ subunits (Nusser et al., 1996). Intriguingly, Pclo $^{g t / g t}$ rats display a reduced expression of the $\mathrm{GABA}_{\mathrm{A}} \alpha 6$ subunit at MFs (Fig. 6). This is in line with findings from Medrano et al. (2019), who performed RNA sequencing on $P c l o^{g t / g t}$ rats and found a severe reduction in $\mathrm{GABA}_{\mathrm{A}} \alpha 6$ subunit gene expression. At present, it is unclear why levels of these subunits are reduced. One possibility is that it reflects a homeostatic change within cerebellar circuitry. This concept is supported by electrophysiological data showing that the input resistance of GCs is higher as are mEPSC frequency and EPSC amplitudes (Fig. 8). This condition might arise to compensate for the smaller MF terminals reaching the GCL, which could also have weaker output properties. Indeed, MF boutons have been shown to reduce functional NMDA receptors in GCs depleted of GABAergic inhibition (Watanabe et al., 1998), demonstrating the plasticity of the MF-GC synapse. Alternatively, reduced $\mathrm{GABA}_{\mathrm{A}} \alpha 6$ expression could be due to lower levels of BDNF at MF terminals (Chen et al., 2016), which is necessary to promote the formation of GABAergic synapses onto GCs. Although a role of Piccolo in the secretion of BDNF has not been investigated, the expression of Bassoon, which shares significant functional redundancy with Piccolo, is linked to presynaptic levels of BDNF (Heyden et al., 2011). In this regard, the remaining Bassoon protein could suppress BDNF secretion in MF terminals, altering maturation of the glomeruli and $\mathrm{GABA}_{\mathrm{A}} \alpha 6$ expression.

A fundamental question raised by the anatomical, morphological, and functional changes within the cerebellum of rats lacking Piccolo is whether these changes affect the functionality of the cerebellum. In behavioral tests, we observed that $P c l o^{g t / g t}$ rats performed worse than both $\mathrm{Pclo}^{w t / w t}$ and $\mathrm{Pclo}^{w t / g t}$ littermates at motor function tasks, highlighting the recessive nature of the behavioral impairments. Our colleagues (Medrano et al., 2019) also observed epileptic seizures, increased aggression, and suppression of mating/copulation behaviors and thus reproduction in $\mathrm{Pclo}^{g t / g t}$ rats.

In an earlier study, a mouse model lacking primarily the largest Piccolo isoforms is also smaller than WT counterparts, but no epileptic activity or changes in brain anatomy were reported (Mukherjee et al., 2010), implying that the isoforms responsible for the $\mathrm{PCH} 3$ phenotype may still have been present in the Piccolo KO mouse. The broad range of phenotypes and potential downstream effects of Piccolo LOF in the Pclo ${ }^{g t / g t}$ rat highlight the importance to use mutations more closely resembling the single nucleotide polymorphism from $\mathrm{PCH} 3$ patients in future studies.

Together, these data support the concept that Piccolo LOF in patients with $\mathrm{PCH} 3$ could contribute to many of the observed phenotypes, including changes in the volume of brain structures and behavioral abnormalities, such as impaired motor control and epileptic seizures (Rajab et al., 2003; Namavar et al., 2011). With regard to reduced cerebellar function, our studies highlight a prominent role for MF boutons, which are not only smaller in size but with altered synaptic properties. Mechanistic studies, which probe how Piccolo loss contributes to these changes, should provide insights into both the role of Piccolo during cerebellar development and the etiology of PCH3.

\section{References}

Ackermann F, Schink KO, Bruns C, Izsvák Z, Hamra FK, Rosenmund C, Garner CC (2019) Critical role for Piccolo in synaptic vesicle retrieval. Elife 8:e46629.

Ahmad-Annuar A, Ciani L, Simeonidis I, Herreros J, Fredj NB, Rosso SB, Hall A, Brickley S, Salinas PC (2006) Signaling across the synapse: a role for wnt and dishevelled in presynaptic assembly and neurotransmitter release. J Cell Biol 174:127-139.

Ahmed MY, Chioza BA, Rajab A, Schmitz-Abe K, Al-Khayat A, Al-Turki S, Baple EL, Patton MA, Al-Memar AY, Hurles ME, Partlow JN, Hill RS, Evrony GD, Servattalab S, Markianos K, Walsh CA, Crosby AH, Mochida GH (2015) Loss of PCLO function underlies pontocerebellar hypoplasia type III. Neurology 84:1745-1750.

Apps R, Garwicz M (2005) Anatomical and physiological foundations of cerebellar information processing. Nat Rev Neurosci 6:297-311.

Brickley SG, Cull-Candy SG, Farrant M (1996) Development of a tonic form of synaptic inhibition in rat cerebellar granule cells resulting from persistent activation of GABAA receptors. J Physiol 497:753-759.

Byczkowicz N, Ritzau-Jost A, Delvendahl I, Hallermann S (2018) How to maintain active zone integrity during high-frequency transmission. Neurosci Res 127:61-69.

Cases-Langhoff C, Voss B, Garner AM, Appeltauer U, Takei K, Kindler S, Veh RW, De Camilli P, Gundelfinger ED, Garner CC (1996) Piccolo, a novel $420 \mathrm{kDa}$ protein associated with the presynaptic cytomatrix. Eur J Cell Biol 69:214-223.

Chen AI, Zang K, Masliah E, Reichardt LF (2016) Glutamatergic axon- 
derived BDNF controls GABAergic synaptic differentiation in the cerebellum. Sci Rep 6:20201.

Curzon P, Zhang M, Radek RJ, Fox GB (2009) The behavioral assessment of sensorimotor processes in the mouse: acoustic startle, sensory gating, locomotor activity, rotarod, and beam walking. In: Methods of behavior analysis in neuroscience (Buccafusco JJ, ed). Boca Raton, FL: CRC.

Durmaz B, Wollnik B, Cogulu O, Li Y, Tekgul H, Hazan F, Ozkinay F (2009) Pontocerebellar hypoplasia type III (CLAM): extended phenotype and novel molecular findings. J Neurol 256:416-419.

Eccles JC, Llinás R, Sasaki K (1966) The mossy fibre-granule cell relay of the cerebellum and its inhibitory control by Golgi cells. Exp Brain Res $1: 82-101$.

Eshra A, Hirrlinger P, Hallermann S (2019) Enriched environment shortens the duration of action potentials in cerebellar granule cells. Front Cell Neurosci 13:289.

Fenster SD, Garner CC (2002) Gene structure and genetic localization of the PCLO gene encoding the presynaptic active zone protein piccolo. Int J Dev Neurosci 20:161-171.

Fenster SD, Chung WJ, Zhai R, Cases-Langhoff C, Voss B, Garner AM, Kaempf U, Kindler S, Gundelfinger ED, Garner CC (2000) Piccolo, a presynaptic zinc finger protein structurally related to bassoon. Neuron 25:203-214.

Gao C, Chen YG (2010) Dishevelled: the hub of wnt signaling. Cell Signal 22:717-727.

Gundelfinger ED, Reissner C, Garner CC (2015) Role of bassoon and piccolo in assembly and molecular organization of the active zone. Front Synaptic Neurosci 7:19.

Hall AC, Lucas FR, Salinas PC (2000) Axonal remodeling and synaptic differentiation in the cerebellum is regulated by WNT-7a signaling. Cell 100:525-535.

Hashimoto K, Kano M (1998) Presynaptic origin of paired-pulse depression at climbing fibre-Purkinje cell synapses in the rat cerebellum. J Physiol 506:391-405.

Hashimoto K, Ichikawa R, Kitamura K, Watanabe M, Kano M (2009) Translocation of a "winner" climbing fiber to the Purkinje cell dendrite and subsequent elimination of "losers" from the soma in developing cerebellum. Neuron 63:106-118.

Heyden A, Ionescu MC, Romorini S, Kracht B, Ghiglieri V, Calabresi P, Seidenbecher C, Angenstein F, Gundelfinger ED (2011) Hippocampal enlargement in bassoon-mutant mice is associated with enhanced neurogenesis, reduced apoptosis, and abnormal BDNF levels. Cell Tissue Res 346:11-26.

Homanics GE, Ferguson C, Quinlan JJ, Daggett J, Snyder K, Lagenaur C, Mi ZP, Wang XH, Grayson DR, Firestone LL (1997) Gene knockout of the $\alpha 6$ subunit of the $\gamma$-aminobutyric acid type A receptor: lack of effect on responses to ethanol, pentobarbital, and general anesthetics. Mol Pharmacol 51:588-596.

Hübler D, Rankovic M, Richter K, Lazarevic V, Altrock WD, Fischer KD, Gundelfinger ED, Fejtova A (2012) Differential spatial expression and subcellular localization of CtBP family members in rodent brain. PLoS One 7:e39710.

Ichikawa R, Hashimoto K, Miyazaki T, Uchigashima M, Yamasaki M, Aiba A, Kano M, Watanabe M (2016) Territories of heterologous inputs onto Purkinje cell dendrites are segregated by mGluR1-dependent parallel fiber synapse elimination. Proc Natl Acad Sci U S A 113:2282-2287.

Ivanova D, Dirks A, Montenegro-Venegas C, Schöne C, Altrock WD, Marini C, Frischknecht R, Schanze D, Zenker M, Gundelfinger ED, Fejtova A (2015) Synaptic activity controls localization and function of CtBP1 via binding to bassoon and piccolo. EMBO J 34:1056-1077.

Ivics Z, Li MA, Mates L, Boeke JD, Nagy A, Bradley A, Izsvák Z (2009) Transposon-mediated genome manipulation in vertebrates. Nat Methods 6:415-422.

Izsvák Z, Fröhlich J, Grabundzija I, Shirley JR, Powell HM, Chapman KM, Ivics Z, Hamra FK (2010) Generating knockout rats by transposon mutagenesis in spermatogonial stem cells. Nat Methods 7:443-445.

Jakab RL, Hámori J (1988) Quantitative morphology and synaptology of cerebellar glomeruli in the rat. Anat Embryol 179:81-88.

Leto K, Arancillo M, Becker EB, Buffo A, Chiang C, Ding B, Dobyns WB, Dusart I, Haldipur P, Hatten ME, Hoshino M, Joyner AL, Kano M, Kilpatrick DL, Koibuchi N, Marino S, Martinez S, Millen KJ, Millner TO, Miyata T, et al. (2016) Consensus paper: cerebellar development. Cerebellum 15:789-828.
Maex R, De Schutter E (1998) Synchronization of Golgi and granule cell firing in a detailed network model of the cerebellar granule cell layer. J Neurophysiol 80:2521-2537.

Maricich SM, Aqeeb KA, Moayedi Y, Mathes EL, Patel MS, Chitayat D, Lyon G, Leroy JG, Zoghbi HY (2011) Pontocerebellar hypoplasia: review of classification and genetics, and exclusion of several genes known to be important for cerebellar development. J Child Neurol 26:288-294.

Martinez M, Torres VI, Vio CP, Inestrosa NC (2019) Canonical Wnt signaling modulates the expression of pre- and postsynaptic components in different temporal patterns. Mol Neurobiol. Advance online publication. Retrieved Nov 19, 2019. doi: 10.1007/s12035-019-01785-5.

Mayhew TM, Gundersen HJ (1996) If you assume, you can make an ass out of $u$ and me': a decade of the disector for stereological counting of particles in 3D space. J Anat 188:1-15.

Medrano GA, Singh M, Plautz EJ, Good LB, Chapman KM, Chaudhary J, Jaichander P, Powell HM, Pudasaini A, Shelton JM, Richardson JA, Xie XJ, Ivics Z, Braun C, Ackermann F, Garner CC, Izsvák Z, Hamra FK (2019) Mutant screen reveals depression-associated Piccolo's control over brain-gonad cross talk and reproductive behavior. bioRxiv: 405985 .

Metz GA, Whishaw IQ (2009) The ladder rung walking task: a scoring system and its practical application. J Vis Exp 12:1204.

Miyashita S, Adachi T, Yamashita M, Sota T, Hoshino M (2017) Dynamics of the cell division orientation of granule cell precursors during cerebellar development. Mech Dev 147:1-7.

Miyazaki T, Fukaya M, Shimizu H, Watanabe M (2003) Subtype switching of vesicular glutamate transporters at parallel fibre-Purkinje cell synapses in developing mouse cerebellum. Eur J Neurosci 17:2563-2572.

Mukherjee K, Yang X, Gerber SH, Kwon HB, Ho A, Castillo PE, Liu X, Südhof TC (2010) Piccolo and bassoon maintain synaptic vesicle clustering without directly participating in vesicle exocytosis. Proc Natl Acad Sci U S A 107:6504-6509.

Müller TM, Gierke K, Joachimsthaler A, Sticht H, Izsvák Z, Hamra FK, Fejtová A, Ackermann F, Garner CC, Kremers J, Brandstätter JH, RegusLeidig H (2019) A multiple piccolino-RIBEYE interaction supports plate-shaped synaptic ribbons in retinal neurons. J Neurosci 39:26062619.

Namavar Y, Barth PG, Kasher PR, van Ruissen F, Brockmann K, Bernert G, Writzl K, Ventura K, Cheng EY, Ferriero DM, Basel-Vanagaite L, Eggens VR, Krägeloh-Mann I, De Meirleir L, King M, Graham JM Jr, von Moers A, Knoers N, Sztriha L, Korinthenberg R, et al. (2011) Clinical, neuroradiological and genetic findings in pontocerebellar hypoplasia. Brain 134:143-156.

Nusser Z, Sieghart W, Stephenson FA, Somogyi P (1996) The alpha 6 subunit of the GABAA receptor is concentrated in both inhibitory and excitatory synapses on cerebellar granule cells. J Neurosci 16:103-114.

Nusser Z, Sieghart W, Somogyi P (1998) Segregation of different GABAA receptors to synaptic and extrasynaptic membranes of cerebellar granule cells. J Neurosci 18:1693-1703.

Rajab A, Mochida GH, Hill A, Ganesh V, Bodell A, Riaz A, Grant PE, Shugart YY, Walsh CA (2003) A novel form of pontocerebellar hypoplasia maps to chromosome 7q11-21. Neurology 60:1664-1667.

Regus-Leidig H, Fuchs M, Löhner M, Leist SR, Leal-Ortiz S, Chiodo VA, Hauswirth WW, Garner CC, Brandstätter JH (2014) In vivo knockdown of piccolino disrupts presynaptic ribbon morphology in mouse photoreceptor synapses. Front Cell Neurosci 8:259.

Rothman JS, Silver RA (2018) NeuroMatic: an integrated open-source software toolkit for acquisition, analysis and simulation of electrophysiological data. Front Neuroinform 12:14.

Rudnik-Schöneborn S, Barth PG, Zerres K (2014) Pontocerebellar hypoplasia. Am J Med Genet C Semin Med Genet 166C:173-183.

Salinas PC, Zou Y (2008) Wnt signaling in neural circuit assembly. Annu Rev Neurosci 31:339-358.

Scheiffele P (2003) Cell-cell signaling during synapse formation in the CNS. Annu Rev Neurosci 26:485-508.

Schindelin J, Arganda-Carreras I, Frise E, Kaynig V, Longair M, Pietzsch T, Preibisch S, Rueden C, Saalfeld S, Schmid B, Tinevez JY, White DJ, Hartenstein V, Eliceiri K, Tomancak P, Cardona A (2012) Fiji: an opensource platform for biological-image analysis. Nat Methods 9:676-682.

Shinoda Y, Sugiuchi Y, Futami T, Izawa R (1992) Axon collaterals of mossy fibers from the pontine nucleus in the cerebellar dentate nucleus. J Neurophysiol 67:547-560. 
Sillitoe R, Fu Y, Watson, C (2012) Cerebellum. San Diego: Academic.

Solecki DJ, Liu XL, Tomoda T, Fang Y, Hatten ME (2001) Activated Notch2 signaling inhibits differentiation of cerebellar granule neuron precursors by maintaining proliferation. Neuron 31:557-568.

Turrigiano G (2012) Homeostatic synaptic plasticity: local and global mechanisms for stabilizing neuronal function. Cold Spring Harb Perspect Biol 4:a005736.

Uhlén M, Fagerberg L, Hallström BM, Lindskog C, Oksvold P, Mardinoglu A, Sivertsson Å, Kampf C, Sjöstedt E, Asplund A, Olsson I, Edlund K, Lundberg E, Navani S, Szigyarto CA, Odeberg J, Djureinovic D, Takanen JO, Hober S, Alm T, et al. (2015) The human protein atlas. Science 347:1260419.

Voogd J, Glickstein M (1998) The anatomy of the cerebellum. Trends Neurosci 21:370-375.

Wagh D, Terry-Lorenzo R, Waites CL, Leal-Ortiz SA, Maas C, Reimer RJ, Garner CC (2015) Piccolo directs activity dependent F-actin assembly from presynaptic active zones via Daam1. PLoS One 10:e0120093.

Waites CL, Craig AM, Garner CC (2005) Mechanisms of vertebrate synaptogenesis. Annu Rev Neurosci 28:251-274.

Waites CL, Leal-Ortiz SA, Okerlund N, Dalke H, Fejtova A, Altrock WD, Gundelfinger ED, Garner CC (2013) Bassoon and piccolo maintain syn- apse integrity by regulating protein ubiquitination and degradation. EMBO J 32:954-969.

Wallace VA (1999) Purkinje-cell-derived sonic hedgehog regulates granule neuron precursor cell proliferation in the developing mouse cerebellum. Curr Biol 9:445-448.

Watanabe D, Inokawa H, Hashimoto K, Suzuki N, Kano M, Shigemoto R, Hirano T, Toyama K, Kaneko S, Yokoi M, Moriyoshi K, Suzuki M, Kobayashi K, Nagatsu T, Kreitman RJ, Pastan I, Nakanishi S (1998) Ablation of cerebellar Golgi cells disrupts synaptic integration involving GABA inhibition and NMDA receptor activation in motor coordination. Cell 95:17-27.

Xu-Friedman MA, Regehr WG (2003) Ultrastructural contributions to desensitization at cerebellar mossy fiber to granule cell synapses. J Neurosci 23:2182-2192.

Zelnik N, Dobyns WB, Forem SL, Kolodny EH (1996) Congenital pontocerebellar atrophy in three patients: clinical, radiologic and etiologic considerations. Neuroradiology 38:684-687.

Zhai RG, Vardinon-Friedman H, Cases-Langhoff C, Becker B, Gundelfinger ED, Ziv NE, Garner CC (2001) Assembling the presynaptic active zone: a characterization of an active one precursor vesicle. Neuron 29:131-143. 